\title{
Modeling Risk Premia in Forward Foreign Exchange Rates as Unobserved Components: The Model Identification Problem
}

\author{
Aziz Chouikh ${ }^{1} \&$ Abdelwahed Trabelsi ${ }^{2}$ \\ ${ }^{1}$ Department of Finance, Institut Supérieur d'Informatique et de Gestion, University of Kairouan, Kairouan, Tunisia \\ ${ }^{2}$ Department of Statistics, Institut Supérieur de Gestion, University of Tunis, Tunis, Tunisia \\ Correspondence: Aziz Chouikh, Institut Supérieur d'Informatique et de Gestion, University of Kairouan, Avenue \\ Khmaies El Alouini, 3100, Kairouan, Tunisia. Tel: 216-7-723-6571. E-mail: azizchouikh@gmail.com
}

Received: March 19, 2014

doi:10.5430/ijfr.v5n3p119
Accepted: April 11, $2014 \quad$ Online Published: June 18, 2014

URL: http://dx.doi.org/10.5430/ijfr.v5n3p119

\begin{abstract}
Is an autoregressive moving average model for the unobserved forward risk premium component always identifiable? Is the signal extraction-based approach always feasible? In this paper, we point out a theoretical framework to shed the light on the statistical problem of model identification. We find out that whenever a model for the unobservable forward risk premium is unidentifiable, we identify a new class of functions that we call: the noise generating functions (Hereafter NGF). These functions circumvent the model identification problem and help us make insights on the noise variances. We demonstrate that a model for the risk premium in the forward exchange rate is not always identifiable and the signal extraction methodology is not always feasible. In addition, our theoretical statements are applied to the empirically evidenced models within the related literature.
\end{abstract}

Keywords: autoregressive moving average, forward risk premium, signal extraction, noise, forward exchange rate

\section{Introduction}

Not only do forward risk premia evoke much debate but also they are central to the theory in/of international finance. To make insights on modeling the forward risk premium component in the forward exchange rate is sine qua non for understanding its behavior and predicting it. In addition, the enlightenment of the labyrinthine clues of the forward premium puzzle (Note 1) makes easier the implementation of adequate policy tools for Central Banks. A huge body of the empirical literature has been documenting many forward risk premium component modeling approaches. In fact, continuous time vs discrete time models, linear vs nonlinear models, parametric vs nonparametric models, observed vs unobserved factor models, and regression-based vs signal extraction-based models have been performed. Engel (1996) surveyed several techniques of modeling and testing forward risk premium features such that the consumption capital asset pricing model, the latent variable model and portfolio-balance models. Diko, Lawford and Limpens (2006) investigated the presence of electricity forward risk premia in a continuous time framework and using an unobserved factor model. They adopted nonlinear and nonparametric estimation techniques. Bernoth, Von Hagen and De Vries (2010) performed an unobserved factor model to futures exchange rates. Fama (1984) implemented a regression-based approach where the forward risk premium is unobserved but it is explained via observed variables, however Wolff (1987) set up the signal extraction-based approach wherein the forward risk premium is modeled as an unobserved component. Also Cheung (1993) modeled risk premia in forward exchange rates as unobservables and pointed out a signal extraction modeling strategy. Bhar and Chiarella (2009) compared the signal extraction approach in continuous-time settings and discrete-time settings of forward risk premia. Rezessy (2010) applied three approaches from which the signal extraction approach and made a crosscheck based on them. Moreover, Cavaglia, Verschoor and Wolff (1994) pointed out, using a survey forecast data, a direct measurement of the forward risk premium and so it becomes observable. On the other hand, Bidarkota (2004) found out that the signal plus noise model failed to isolate statistically significant risk premium components from the noise. Furthermore, Jacobs (1982), Boyer and Adams (1988) and Bekaert and Hodrick (1993) shed the light on the measurement errors, model misspecifications and errors-in-variables problems whenever the regression-based approach is carried out. Gospodinov (2009) argued that the widely reported empirical literature of regressing the future exchange return on the current forward premium evidences several econometric limits that should be alleviated. In addition, Djeutem (2013) stated that the forward premium puzzle, in a context wherein agents doubt the 
specification of their models, is explained by a model misspecification.

Within the growing body of the empirical literature, when the financial researcher decides to handle with the forward premium anomaly, he has to choose between modeling the forward risk premium components as observables or unobservables. The former case is veracious if and only if we observe the conditional expectation of the future spot exchange rate. Indeed, Nijman, Palm and Wolff (1993) pointed out two conditions that must be satisfied to make the forward risk premium be an observed component. Once these two conditions are satisfied, the forward risk premium becomes observable and equals the forward premium. These conditions are: first, the spot exchange rate pursues a random walk stochastic process (Note 2) then the conditional appreciation/depreciation is equal to zero. Second, the semi-strong market efficiency (event studies) coincides with the weak-form market efficiency (tests for return forecastability) (Note 3). It is obvious that the required assumptions to get the forward risk premium observed are strongly restrictive. Thereby we dismiss this case. When we decide to model the forward exchange risk premium as unobservable, we have to choose between explaining it via observed variables and directly modeling it as unobservable. To study the time variation in premia and other features, we can set up either a regression-based modeling strategy or a signal-extraction modeling strategy. As mentioned by Wolff (1987), the regression-based approach has shortcomings and depends on the researcher's choice of dependent (endogenous) and independent (exogenous) variables. This arbitrariness is also dictated by the availability of the underlying data. On the other hand, the signal extraction-based approach circumvents the problem of arbitrariness of the traders'information set and it models the risk premium component as a whole, at the expense of identifying an exact econometric model for the signal. Thus, we alleviate the problem of modeling either the systematic risk or the individual relative risk aversion as a constant or a time-varying parameter.

The pertaining related literature, which considers the forward risk premium as unobservable, does not precise whether the hypothesized model for the unobserved forward risk premium component is identifiable or not and does not analyse the case wherein the model is unidentifiable. Furthermore, the previous empirical literature did not emphasize the statistical problem of model identification. In this paper, we aim to answer the following questions: Is an autoregressive moving average (Hereafter ARMA) model for the unobserved forward risk premium component always identifiable? If not, what are the underlying implications?

The remainder of the paper is organized as follows. Section 2 sets up the signal extraction preliminaries. Section 3 points out general autoregressive (AR) and moving average (MA) order conditions for unobserved stochastic processes. Section 4 implements a theoretical framework. Section 5 apllies theoretical issues to previously evidenced models in the literature. Section 6 concludes.

\section{The Signal Extraction Preliminaries}

The signal extraction methodology emanates from the engineering branch. It consists of writing a model in a state-space form (SSF) and applying the Kalman Filter (KF). A SSF deals with two equations: a measurement equation and a transition or state equation. The measurement equation is also called the signal plus noise model. It involves an observed time-series as a sum of two unobserved components: the signal and the noise. The signal or the unobserved state variable is deemed as buried in the noisy environment. It is a kind of interference between the signal and the noise. Thus the former should be extracted from the latter. To do so, a state equation which describes the signal stochastic process has to be specified and a filtering algorithm has to be run. The KF aims to isolate the unobservable signal from the unobservable noise. The signal extraction methodology is as follws: (a) to identify an ARMA model for the observable time-series, (b) to make an assumption on the noise stochastic ARMA process, (c) to infer an ARMA model for the unobservable signal using Ansley, Spivey and Wrobleski (Hereafter ASW) (1977)'s summation theorem (Note 4) of moving average processes, (d) to derive a SSF, and (e) to set up the KF.

\subsection{The Signal plus Noise Model}

Our starting point is the Fama (1984)'s definition of the forward exchange rate: the forward foreign exchange rate at time $t$ for a delivery at time $t+1$ is the market determined certainty equivalent (Note 5 ). Therefore, it is the sum of the Markowitz forward risk premium, at time $t$, and the conditional expectation, made at time $t$, of the future spot exchange rate at time $\mathrm{t}+1$. It follows:

$$
f_{t, 1, i}=P_{t, 1, i}+E\left(S_{t+1, i} / \Psi_{t}\right)
$$

Where $f_{t, 1, i}$ is the $\mathrm{i}^{\text {th }}$ forward exchange rate at time $\mathrm{t}$ for a delivery at time $\mathrm{t}+1, P_{t, 1, i}$ is the $\mathrm{i}^{\text {th }}$ one-period unobservable forward risk premium and $E\left(S_{t+1, i} / \Psi_{t}\right)$ is the unobservable conditional expectation of the $i^{\text {th }}$ future spot exchange rate. $\Psi_{t}$ is the information set on which we form the conditional expectation. Substracting the realized future spot exchange rate from both sides of equation (1), we get: 


$$
f_{t, 1, i}-S_{t+1, i}=P_{t, 1, i}+E\left(S_{t+1, i} / \Psi_{t}\right)-S_{t+1, i}
$$

Let $f_{t, 1, i}-S_{t+1, i}=y_{t+1, i}$ be the forecast error of using the forward exchange rate as a predictor of the future spot exchange rate. $y_{t+1, i}$ can also be interpreted as the gross return corresponding to selling (buying) forward at time $t$ a unit of the $i^{\text {th }}$ foreign currency for a delivery at time $\mathrm{t}+1$ and buying (selling) spot at $\mathrm{t}+1 . y_{t+1, i}$ is observed at time $\mathrm{t}+1$. Let $E\left(S_{t+1, i} / \Psi_{t}\right)-S_{t+1, i}=\varepsilon_{t+1, i}$ be the forecast error due to information arrivals and market imperfections. Rewriting equation (2), we get:

$$
y_{t+1, i}=P_{t, 1, i}+\varepsilon_{t+1, i}
$$

Only the left-hand side, of equation (3), is observable at time $t+1$. Both the forward risk premium, $P_{t, 1, i}$, and the forecast error, $\varepsilon_{t+1, i}$, are unobservable but we only observe their sum. It can be easily seen that $\varepsilon_{t+1, i}$ is a noise. $P_{t, 1, i}$ is the fundamental signal buried in the noise $\varepsilon_{t+1, i}$. It is ipso facto deduced that equation (3) is the signal plus noise equation which is also called the measurement equation and it is the cornerstone of the structural form (Hereafter SF). The signal $P_{t, 1, i}$ is the state variable and it is unobserved. In fact, what we have is an observed model that is equal to the sum of two unobserved models. Let $\mathrm{M}_{\mathrm{y}}$ be the observed, i.e. known, model relative to the observed time series. Let $\mathrm{M}_{\text {signal }}$ be the unobserved and unknown model for the signal. Let $\mathrm{M}_{\text {noise }}$ be the unobserved and unknown model for the first source of noise. It follows that $\mathrm{M}_{\mathrm{y}}=\mathrm{M}_{\text {signal }}+\mathrm{M}_{\text {noise. }}$. Knowing the noise model is empirically sine qua non for the application of the ASW's moving average summation theorem. Moreover, we will implement our framework according to ARMA-based modeling.

Assumption 1: ARMA modeling is economically rational in the sense of Feige and Pearce (Note 6).

Assumption 2: $\left(y_{t+1, i}=f_{t, 1, i}-S_{t+1, i}\right)$ follows a causal-invertible $\operatorname{ARMA}\left(p_{1}, q_{1}\right)$ model, $\left(P_{t, 1, i}\right)$ follows a causal-invertible $\operatorname{ARMA}\left(p_{2}, q_{2}\right)$ model, and $\left(\varepsilon_{t+1, i}\right)$ follows a hypothetically known causal-invertible $A R M A\left(p_{3}, q_{3}\right)$ model (Note 7$)$. Common roots are not allowed to exist.

Assumption 3: $\left(P_{t, 1, i}\right)$ and $\left(\varepsilon_{t+1, i}\right)$ have uncorrelated moving average structures (Note 8).

According to assumption 2, we get:

$$
\begin{gathered}
\Phi_{y}(B) y_{t+1, i}=\Theta_{y}(B) \omega_{t+1, i} \\
\Phi_{P}(B) P_{t, 1, i}=\Theta_{P}(B) a_{t, i} \\
\Phi_{\varepsilon}(B) \varepsilon_{t+1, i}=\Theta_{\varepsilon}(B) v_{t+1, i}
\end{gathered}
$$

Where $\Phi(B)$ and $\Theta(B)$ are polynomials in B, the backward shift operator. $\left(\omega_{t+1, i}\right),\left(a_{t, i}\right)$ (Note 9$)$ and $\left(v_{t+1, i}\right)$ are white noise processes.

\subsection{The Model Identification Problem}

Equation (4), called also the reduced form (Hereafter RF), is directly estimated given that $y_{t+1, i}$ is observable, so we identify $\left(p_{1}, q_{1}\right)$, the AR and/or MA coefficient estimates and the variance estimate of $\omega_{t+1, i}, \hat{\sigma}_{\omega, i}^{2}$. Equation (6), given assumption 2 , characterizes a white noise stochastic process, i.e. $\operatorname{ARMA}(0,0)$. As we have mentioned above, we will use the ASW's moving average summation theorem to infer hypothetical models for the signal, $P_{t, 1, i}$. In addition, the signal extraction via the KF is feasible if a SSF is derived and the latter is pointed out if an ARMA model for the signal is identifiable. Otherwise, the signal extraction-based approach is infeasible. The question is: when does a model identification problem arise? The model identification problem arises whenever one of the following items, at least, is not identified: (a) $\left(p_{2}, q_{2}\right)$, (b) AR and/or MA coefficient(s) estimate(s) of the $\operatorname{ARMA}\left(p_{2}, q_{2}\right)$ model, and (c) the variance of the first source of noise, $\sigma_{\varepsilon, i}^{2}$, and/or the variance of the second source of noise, $\sigma_{a, i}^{2}$. We say that there is no identification problem, and therefore the model is identifiable, if and only if all the items cited above are identifiable. If not, the model identification problem does exist.

An intuitive thought is: how do we circumvent an eventual model identification problem? What should we do whether it happens? Nijman, Palm and Wolff (1993), in such a case, looked for upper and lower bounds for the unidentifiable variances $\sigma_{\varepsilon, i}^{2}$ and $\sigma_{a, i}^{2}$. However, they considered a special case and did not generalize. We propose to point out a more general framework wherein we find out more generalized upper and lower bounds.

If we evidence unidentifiable parameters, and so does the model for the unobservable signal, it is obvious that either an underdetermination or an overdetermination does matter. We will shed the light on those cases and their implications.

\section{General AR and MA Order Conditions for Unobserved Stochastic Processes}

In this section, we will set up the general AR and MA order conditions for the unobserved model for the signal. In 
fact, we will answer the following question: knowing the stochastic process of the observable time series of the realized forecasting error of using the forward exchange rate as a predictor of the future spot exchange rate, i.e. $\left(p_{1}, q_{1}\right)$ is known, what conditions should the signal AR and MA orders, $\left(p_{2}, q_{2}\right)$, verify?

Proposition 1: Under assumptions 2 and 3 and using the ASW's moving average summation theorem, we get $\left(p_{2}, q_{2}\right)$ such that: $p_{1}=p_{2}$ and $q_{1} \leq \operatorname{Max}\left(q_{2}, p_{1}\right)$.

Proof: See Appendix 1.

Corollary 1: $\Phi_{y}(B)=\Phi_{P}(B)$

Proof: See Appendix 1.

Proposition 1 states the conditions that the hypothetical models for the unobserved forward risk premium must satisfy. Let $H$ be the set of inferred hypothetical models for the signal, we have: $H=\left\{\operatorname{ARMA}\left(p_{2}, q_{2}\right) / p_{1}=p_{2}\right.$ and $\left.q_{1} \leq \operatorname{Max}\left(q_{2}, p_{1}\right)\right\}$. Obviously, the cardinality of $H$ is greater or equal to one. Let $\mathrm{M}^{*} \mathrm{p}$ be the identifiable $\operatorname{ARMA}\left(p_{2}^{*}, q_{2}^{*}\right)$ model for the signal. What are the existence conditions of $\mathrm{M}^{*}$ ? ? And if it does exist, is it unique? If the existence conditions are unsatisfied, what are the implications?

An identifiable model does not mean a true model. The reader should keep in mind that the identification and the veracity of the model for the unobservable forward risk premium do not match. The identification is sine qua non for the implementation of the KF, however the true model may be unidentifiable. Thus, the class of unidentifiable models for the unobserved signal is our main topic and we will focus on it.

\section{4. $\operatorname{ARMA}\left(\mathrm{p}_{1}, \mathrm{q}_{1}\right)$ for the Observed Time Series and a White Noise Forecast Error}

For the observed time series, $\left(y_{t+1, i}=f_{t, 1, i}-S_{t+1, i}\right)$, an $\operatorname{ARMA}\left(p_{1}, q_{1}\right)$ is unique. The estimated equation (4) points out the RF, while the equation (3) and the explicitely hypothesized model, via the ASW's theorem, for the unobserved forward risk premium component point out the SF. Given the uniqueness of the $\operatorname{ARMA}\left(p_{1}, q_{1}\right)$ representation, the RF and SF associated to the observed time series, $\left(y_{t+1, i}\right)$, generate equal variances and k-order autocovariances. Let $\gamma_{0, R F}=E\left(y_{t+1, i}^{2}\right)$ be the variance of $y_{t+1, i}$ from the RF and let $\gamma_{0, S F}=E\left(y_{t+1, i}^{2}\right)$ be the variance of $y_{t+1, i}$ from the SF. Let $\gamma_{k, R F}=E\left(y_{t+1, i} y_{t+1-k, i}\right)$ be the k-order autocovariance from the RF and let $\gamma_{k, S F}=E\left(y_{t+1, i} y_{t+1-k, i}\right)$ be the k-order autocovariance from the SF (Note 10). It follows $\gamma_{k, R F}=\gamma_{k, S F}$ for all $0 \leq k \leq \operatorname{Max}\left(p_{1}, q_{1}\right)$. Therefore, we get $\operatorname{Max}\left(p_{1}, q_{1}\right)+1$ linearly independent equations (Note 11). On the other hand, it can be easily seen that the only unknown polynomial is $\Theta_{P}(B)$ which consists of $q_{2}$ MA coefficients. Straightforwardly, we deduce that we get $q_{2}+2$ unknowns to identify: $q_{2}$ MA coefficients and the noise variances, $\sigma_{\varepsilon}^{2}$ and $\sigma_{a}^{2}$.

Proposition $2: \mathrm{M}_{\mathrm{P}} \exists$ if and only if $\mathrm{M}_{\mathrm{P}}^{*} \in H$ and $q_{2}^{*}=\operatorname{Max}\left(p_{1}, q_{1}\right)-1$.

Proof: See Appendix 2.

Proposition 2 points out both the identification conditions and the uniqueness of $\mathrm{M}_{\mathrm{p}}$ whenever these conditions hold. Otherwise, a model for the unobservable forward risk premium component is unidentifiable and we deal with either an underdetermination or an overdetermination. The model identification problem takes place whenever the well-known order condition is dissatisfied, i.e. the number of equations and the number of unknowns are unequal.

Proposition 3: If $\mathrm{M}_{\mathrm{P}} \in H$ and $q_{2} \neq \operatorname{Max}\left(p_{1}, q_{1}\right)-1$ then we get, under appropriate conditions, the following two sub-cases: (a) $q_{2}>\operatorname{Max}\left(p_{1}, q_{1}\right)-1$ so infinitely many solutions, and (b) $q_{2}<\operatorname{Max}\left(p_{1}, q_{1}\right)-1$ so no solutions.

Proof: See Appendix 3.

The proposition 3-b reflect an overdetermined system of equations, while proposition 3-a reflects an underdetermination on which we will focus. In fact, according to linear algebra, we can express in such a case of underdetermination, under given conditions, some variables as functions of the remaining ones. In our case, we will try to express noise variances, $\sigma_{\varepsilon, i}^{2}$ and $\sigma_{a, i}^{2}$, as functions of the MA coefficients of the polynomial $\Theta_{P}(B)$. We will call those functions: the NGF. To do so is equivalent to finding out a relationship between the state of the exchange market noise trading, represented by the NGF, and the unknown MA signal coefficients. Moreover, the NGF will let us make inferences about the signal-to-noise ratio, i.e. the variance of the signal divided by the variance of the noise. The model identification problem lets us deal with hypothetical models for the forward risk premium, $\mathrm{M}_{\mathrm{P}}$, which belong to a subset of $H, H_{\mathrm{NGF}}$, such that $H_{\mathrm{NGF}}=\left\{\operatorname{ARMA}\left(p_{2}, q_{2}\right) / p_{1}=p_{2}\right.$ and $q_{1} \leq \operatorname{Max}\left(q_{2}, p_{1}\right)$ and $q_{2}>$ $\left.\operatorname{Max}\left(p_{1}, q_{1}\right)-1\right\}$. If $\mathrm{M}_{\mathrm{P}} \in H_{\mathrm{NGF}}$, it gives rise to NGF.

The moral of the story is whenever a model for the unobserved forward risk premium is unidentifiable and the proposition 3-a holds, we identify the so-called NGF. The latter must: (a) be positive, (b) converge, (c) be upwardly 
and downwardly bounded, and (d) be a bijective function.

Empirically, the observed time series, $\left(y_{t+1, i}=P_{t, 1, i}+\varepsilon_{t+1, i}=f_{t, 1, i}-S_{t+1, i}\right)$, can evidence an $\operatorname{ARMA}\left(p_{1} \geq\right.$ $\left.1, q_{1} \geq 1\right)$ or a pure $M A\left(q_{1} \geq 1\right)$ or a pure $A R\left(p_{1} \geq 1\right)$. It is pedagogically convenient to deal with the last two cases separately.

\subsection{The Case of a $M A\left(q_{1} \geq 1\right)$ for the Observed Time Series and a White Noise Forecast Error}

Proposition 4: If $\left(y_{t+1, i}=f_{t, 1, i}-S_{t+1, i}\right)$ follows an invertible $\mathrm{MA}\left(\mathrm{q}_{1} \geq 1\right)$ stochastic process and $\left(\varepsilon_{t+1, i}\right)$ follows a white noise stochastic process then $\left(P_{t, 1, i}\right)$ follows a hypothetically invertible $\mathrm{MA}\left(\mathrm{q}_{2} \geq \mathrm{q}_{1}\right)$.

Proof: See Appendix 4.

Ipso facto, we deduce that $\mathrm{M}_{\mathrm{p}}^{*}$ does not exist because we always get a number of unknowns greater than the number of equations, i.e. an underdetermined system of equations. Consequently, we have solely hypothetical models for the unobservable signal which belong to $H_{\mathrm{NGF}}$. Neither the identifiable model nor the models causing an overdetermination exist. We can express the proposition 4 in the following table below. We easily show that whenever the observed time series follows an $\operatorname{ARMA}\left(p_{1} \geq 1, q_{1} \geq 1\right)$ such that $p_{1}<q_{1}$, the identifiable model does not exist too.

Table 1. The existence of $\mathrm{M}_{\mathrm{P}}^{*}$ and NGF

\begin{tabular}{ccccc}
\hline Observed Model & $\begin{array}{c}\text { Hypothetical } \\
\text { Models }\end{array}$ & $\mathrm{M}_{\mathrm{p}}$ & $\mathrm{NGF}$ & Overdetermination \\
\hline $\mathrm{MA}\left(\mathrm{q}_{1} \geq 1\right)$ & $\mathrm{MA}\left(\mathrm{q}_{2} \geq \mathrm{q}_{1}\right)$ & Does not exist & Does exist & Does not exist \\
\hline
\end{tabular}

In this case, the SSF is underived, and so does the KF. Therefore, Wolff (1987)'s estimation of the MA(1) model is mathematically infeasible and is only a filtering approximation. As shown in Table 1 above, whenever the observed time series follows a pure moving average process, the signal extraction-based approach is infeasible.

\subsection{The Case of an $A R\left(p_{1} \geq 1\right)$ for the Observed Time Series and a White Noise Forecast Error}

Proposition 5: If $\left(y_{t+1, i}\right)$ follows a causal $\operatorname{AR}\left(\mathrm{p}_{1} \geq 1\right)$ stochastic process and $\left(\varepsilon_{t+1, i}\right)$ follows a white noise stochastic process then $\left(P_{t, 1, i}\right)$ follows a hypothetically causal-invertible $\operatorname{ARMA}\left(\mathrm{p}_{1} \geq 1, \mathrm{q}_{2} \geq 0\right)$.

Proof: See Appendix 5.

Table 2. The existence of $\mathrm{M}_{\mathrm{P}}{ }_{\mathrm{p}}$ and NGF

\begin{tabular}{clll}
\hline & \multicolumn{1}{c}{$\mathrm{p}_{1}=1$} & \multicolumn{1}{c}{$\mathrm{p}_{1}=2$} & \multicolumn{1}{c}{$\mathrm{p}_{1} \geq 3$} \\
\hline$q_{2}=\mathrm{p}_{1}-1$ & $q_{2}=0$ so $\mathrm{M}_{\mathrm{P}}^{*} \exists$ & $q_{2}=1$ so $\mathrm{M}_{\mathrm{p}}^{*} \exists$ & $q_{2}=\mathrm{p}_{1}-1$ so $\mathrm{M}_{\mathrm{P}} \exists$ \\
$q_{2}>\mathrm{p}_{1}-1$ & $q_{2}>0$ so $\mathrm{M}_{\mathrm{P}} \nexists$ and NGF $\exists$ & $q_{2}>1$ so $\mathrm{M}_{\mathrm{p}} \nexists$ and NGF $\exists$ & $q_{2}>\mathrm{p}_{1}-1$ so $\mathrm{M}_{\mathrm{P}} \nexists$ and \\
& & & NGF $\exists$ \\
$q_{2}<\mathrm{p}_{1}-1$ & $q_{2}<0$ absurd & $q_{2}<1 \Rightarrow q_{2}=0$ so neither & $q_{2}<\mathrm{p}_{1}-1$ so $\mathrm{p}_{1}-1$ cases \\
& & $\mathrm{M}_{\mathrm{p}}^{*}$ nor NGF & we get neither $\mathrm{M}_{\mathrm{p}}$ nor NGF \\
\hline
\end{tabular}

As shown in Table 2 above, whenever our aim is to apply the signal estraction-based approach, we have to hypothesize a model for the signal satisfying $q_{2}=\mathrm{p}_{1}-1$. Whenever we aim to point out the corresponding NGF, we undertake a hypothetical model for the signal verifying $q_{2}>p_{1}-1$ as our object of study.

\section{Empirical Issues}

\subsection{Methodology}

Our methodology consists of three steps: (a) we consider the observed time series $\left(y_{t+1, i}=f_{t, 1, i}-S_{t+1, i}\right)$, documented in the empirical literature, (b) we infer $H$, and (c) we point out the eventual $\mathrm{M}^{*}$, the eventual $H_{N G F}$ and whether the overdetermination case exists or not.

We emphasize the study of the observed time series models which are reported in the empirical literature. Reviewing the empirically huge body of the related literature, we carry out the following recap in Table 3 below. 
Table 3. Observable models in the related literature

\begin{tabular}{ccc}
\hline Author(s) & $\begin{array}{c}\text { Observable model for }\left(y_{t+1, i}=\right. \\
\left.f_{t, 1, i}-S_{t+1, i}\right)\end{array}$ & The model for the forecast error \\
\hline Wolff (1987) & $A R \mathrm{MA}(1,1), A R(1)$, and $\mathrm{MA}(1)$ & White noise \\
Cheung (1993) & $A R \mathrm{MA}\left(\mathrm{p}_{1}, q_{1}\right), A R\left(\mathrm{p}_{1}\right)$, and $\mathrm{MA}\left(q_{1}\right) ;$ & White noise \\
& $\mathrm{p}_{1}, q_{1}=1,2$ & \\
Nijman, Palm, and Wolff (1993) & $A R \mathrm{MA}(1,1)$ & White noise \\
Wolff (2000) & $A R \mathrm{MA}(1,1)$ and MA(1). & White noise \\
Bidarkota (2004) & $A R \mathrm{MA}(1,1)$ and $A R(1)$ & White noise \\
Rezessy (2010) & $A R \mathrm{MA}(1,2)$ & MA(2)
\end{tabular}

Based on the underlying recapitulation above, we focus on each case and point out the corresponding NGF. Obviously, the cardinality of $H_{\mathrm{NGF}}$ is greater than or equal to one. Thus, we will focus only on one model, for each case, leading to an underdetermination.

\subsection{The NGFand Their Implications}

For each previously cited empirical model, we highlight its corresponding NGF. The following Table 4 reports all observed models mentioned in Table 3 and gives for each one $H, \mathrm{M}_{\mathrm{p}}^{*}, H_{\mathrm{NGF}}$, and hypothetical model(s) causing overdetermination.

Table 4. Observable models and their corresponding $H_{N G F}$

\begin{tabular}{|c|c|c|c|c|}
\hline $\begin{array}{c}\text { Observable } \\
\text { model for } \\
\left(y_{t+1, i}=\right. \\
\left.f_{t, 1, i}-S_{t+1, i}\right)\end{array}$ & $\begin{array}{l}H: \text { The set of hypothetical models } \\
\text { for the signal }\end{array}$ & $\begin{array}{l}\mathrm{M}_{\mathrm{p}}^{*_{\mathrm{p}} \text { The }} \\
\text { identifiable } \\
\text { model for the } \\
\text { signal }\end{array}$ & $\begin{array}{c}H_{\mathrm{NGF}}: \text { The set of } \\
\text { hypothetical models for } \\
\text { the signal giving rise to } \\
\text { NGF }\end{array}$ & $\begin{array}{c}\text { Hypothetical } \\
\text { Model causing } \\
\text { overdetermina } \\
\text { tion }\end{array}$ \\
\hline$A R \mathrm{MA}(1,1)$ & $\left\{\operatorname{ARMA}\left(1, q_{2}\right) / 1 \leq \operatorname{Max}\left(q_{2}, 1\right)\right\}$ & $A R(1)$ & $\left\{A R M A\left(1, q_{2}\right) / q_{2} \geq 1\right\}$ & $\nexists$ \\
\hline$A R \mathrm{MA}(1,2)$ & $\left\{\operatorname{ARMA}\left(1, q_{2}\right) / 2 \leq \operatorname{Max}\left(q_{2}, 1\right)\right\}$ & $\nexists$ & $\left\{A R M A\left(1, q_{2}\right) / q_{2} \geq 2\right\}$ & $\nexists$ \\
\hline$A R \mathrm{MA}(2,1)$ & $\left\{\operatorname{ARMA}\left(2, q_{2}\right) / 1 \leq \operatorname{Max}\left(q_{2}, 2\right)\right\}$ & $A R \mathrm{MA}(2,1)$ & $\left\{A R M A\left(2, q_{2}\right) / q_{2} \geq 2\right\}$ & $A R(2)$ \\
\hline$A R \mathrm{MA}(2,2)$ & $\left\{\operatorname{ARMA}\left(2, q_{2}\right) / 2 \leq \operatorname{Max}\left(q_{2}, 2\right)\right\}$ & $A R \mathrm{MA}(2,1)$ & $\left\{\operatorname{ARMA}\left(2, q_{2}\right) / q_{2} \geq 2\right\}$ & $A R(2)$ \\
\hline$A R(1)$ & $\left\{\operatorname{ARMA}\left(1, q_{2}\right) / 0 \leq \operatorname{Max}\left(q_{2}, 1\right)\right\}$ & $A R(1)$ & $\left\{A R M A\left(1, q_{2}\right) / q_{2} \geq 1\right\}$ & $\nexists$ \\
\hline$A R(2)$ & $\left\{\operatorname{ARMA}\left(2, q_{2}\right) / 0 \leq \operatorname{Max}\left(q_{2}, 2\right)\right\}$ & $A R \mathrm{MA}(2,1)$ & $\left\{\operatorname{ARMA}\left(2, q_{2}\right) / q_{2} \geq 2\right\}$ & $A R(2)$ \\
\hline $\mathrm{MA}(1)$ & $\left\{M A\left(q_{2}\right) / 1 \leq \operatorname{Max}\left(q_{2}, 0\right)\right\}$ & $\nexists$ & $\left\{M A\left(q_{2}\right) / q_{2} \geq 1\right\}$ & $\nexists$ \\
\hline $\mathrm{MA}(2)$ & $\left\{M A\left(q_{2}\right) / 2 \leq \operatorname{Max}\left(q_{2}, 0\right)\right\}$ & $\nexists$ & $\left\{M A\left(q_{2}\right) / q_{2} \geq 2\right\}$ & $\nexists$ \\
\hline
\end{tabular}

We will concentrate on three categories of ARMA models: mixed ARMA processes, pure AR processes, and pure MA processes for the observable time series, $\left(y_{t+1, i}=f_{t, 1, i}-S_{t+1, i}\right)$. From each category, we will deal with two models. The rationale behind this choice is that each category will deal with two models leading to two systems of equations: a system with two equations and three unknowns and a system with three equations and four unknowns. Moreover, our choice encompasses the most evidenced models for the observed time series.

5.2.1 $\operatorname{ARMA}(1,1)$ as an Observable Model and $\operatorname{ARMA}(1,1)$ as a Hypothetical Model for the Signal

$\left(y_{t+1, i}=f_{t, 1, i}-S_{t+1, i}\right)$ follows an observable $\operatorname{ARMA}(1,1)$ model then we get the following RF:

$$
\left(1-\hat{\phi}_{y, i} B\right) y_{t+1, i}=\left(1-\hat{\theta}_{y, i} B\right) \omega_{t+1, i} \text { (Note 12) }
$$

The forward risk premium hypothetically follows an $A R M A(1,1)$ model then we obtain the following SF:

$$
\left(1-\hat{\phi}_{y, i} B\right) y_{t+1, i}=\left(1-\theta_{P, i} B\right) a_{t, i}+\left(1-\hat{\phi}_{y, i} B\right) \varepsilon_{t+1, i}
$$

Now we calculate the unconditional variance and the first-order autocovariance for both the RF and SF and we get the following system of two equations and three unknowns:

System:

$$
\begin{aligned}
& \gamma_{0, S F}=\gamma_{0, R F} \\
& \gamma_{1, S F}=\gamma_{1, R F}
\end{aligned}
$$




$$
\begin{gathered}
\quad\left(\theta_{P, i}^{2}-2 \hat{\phi}_{y, i} \theta_{P, i}+1\right) \sigma_{a, i}^{2}+\left(1-\hat{\phi}_{y, i}^{2}\right) \sigma_{\varepsilon, i}^{2}=\left(\hat{\theta}_{y, i}^{2}-2 \hat{\phi}_{y, i} \hat{\theta}_{y, i}+1\right) \hat{\sigma}_{\omega, i}^{2} \\
\theta_{P, i} \sigma_{a, i}^{2}+\hat{\phi}_{y, i} \sigma_{\varepsilon, i}^{2}=\hat{\theta}_{y, i} \hat{\sigma}_{\omega, i}^{2}
\end{gathered}
$$

The system above can be rewritten in a matrix form. We have:

$$
X_{\theta_{P, i}} \Sigma_{N G F}=\hat{V}
$$

Where $X_{\theta_{P, i}}$ is a $2 \times 2$ matrix, $\Sigma_{N G F}$ is the $2 \times 1$ NGF vector, and $\hat{V}$ is a known $2 \times 1$ vector such that:

$$
X_{\theta_{P, i}}=\left(\begin{array}{cc}
\theta_{P, i}^{2}-2 \hat{\phi}_{y, i} \theta_{P, i}+1 & 1-\hat{\phi}_{y, i}^{2} \\
\theta_{P, i} & \hat{\phi}_{y, i}
\end{array}\right) ; \Sigma_{N G F}=\left(\begin{array}{c}
\sigma_{a, i}^{2} \\
\sigma_{\varepsilon, i}^{2}
\end{array}\right) \text { and } \hat{V}=\left(\begin{array}{c}
\hat{V}_{1} \\
\hat{V}_{2}
\end{array}\right)=\left(\begin{array}{c}
\left(\hat{\theta}_{y, i}^{2}-2 \hat{\phi}_{y, i} \hat{\theta}_{y, i}+1\right) \hat{\sigma}_{\omega, i}^{2} \\
\hat{\theta}_{y, i} \hat{\sigma}_{\omega, i}^{2}
\end{array}\right) \text {. }
$$

Pointing out the corresponding NGF is equivalent to solving equation (11) in $\Sigma_{\mathrm{NGF}}$. In fact, the eventual solution depends on the invertibility of the matrix $X_{\theta_{P, i}}$ and the conditions, mentioned in section 4, the NGF have to satisfy.

Proposition 6: $\left.\forall \theta_{P, i} \in D_{\theta_{P, i}}=D_{d e t, \theta_{P, i}} \cup D_{h, \theta_{P, i}} ; D_{\text {det, } \theta_{P, i}}=\right]-1, \hat{\phi}_{y, i}[\mathrm{U}] \hat{\phi}_{y, i}, 1\left[\right.$ and $\left.D_{h, \theta_{P, i}}=\right]-1, \hat{\theta}_{y, i}[\mathrm{U}] \hat{\theta}_{y, i}, 1[$ : We have: $\operatorname{det}\left(X_{\theta_{P, i}}\right)=d_{\widehat{\phi}_{y, i}}\left(\theta_{P, i}\right)=\hat{\phi}_{y, i} \theta_{P, i}^{2}-\left(1+\widehat{\phi}_{y, i}^{2}\right) \theta_{P, i}+\widehat{\phi}_{y, i} \neq 0$ then we get:

$$
\Sigma_{N G F}\left(\theta_{P, i}\right)=X_{\theta_{P, i}}^{-1} \hat{V}=\left(\begin{array}{c}
\sigma_{a, i}^{2}\left(\theta_{P, i}\right)=\frac{\hat{A}}{d_{\widehat{\phi}_{y, i}}\left(\theta_{P, i}\right)} \\
\sigma_{\varepsilon, i}^{2}\left(\theta_{P, i}\right)=\hat{\sigma}_{\omega, i}^{2} \frac{h_{\widehat{\theta}_{y, i}}\left(\theta_{P, i}\right)}{d_{\widehat{\phi}_{y, i}}\left(\theta_{P, i}\right)}
\end{array}\right) .
$$

Where $h_{\hat{\theta}_{y, i}}\left(\theta_{P, i}\right)=\hat{\theta}_{y, i} \theta_{P, i}^{2}-\left(1+\hat{\theta}_{y, i}\right) \theta_{P, i}+\hat{\theta}_{y, i}$ and $\hat{A}=\hat{\phi}_{y, i} \hat{V}_{1}+\left(\hat{\phi}_{y, i}^{2}-1\right) \hat{V}_{2}$.

Proof: See Appendix 6.

Proposition 7: Given that $\sigma_{a, i}^{2}\left(\theta_{P, i}\right)$ and $\sigma_{\varepsilon, i}^{2}\left(\theta_{P, i}\right)$ have to be positive, we have:

7-1: $-1<\hat{\theta}_{y, i}<\hat{\phi}_{y, i}<0$ and $\left.\theta_{P, i} \in D_{\theta_{P, i}}=\right]-1, \hat{\theta}_{y, i}[$.

7-2: $-1<\hat{\phi}_{y, i}<\hat{\theta}_{y, i}<0$ or $-1<\hat{\phi}_{y, i}<0<\hat{\theta}_{y, i}<1$ and $\left.\theta_{P, i} \in D_{\theta_{P, i}}=\right] \hat{\theta}_{y, i}, 1[$.

7-3: $0<\hat{\theta}_{y, i}<\hat{\phi}_{y, i}<1$ or $-1<\hat{\theta}_{y, i}<0<\hat{\phi}_{y, i}<1$ and $\left.\theta_{P, i} \in D_{\theta_{P, i}}=\right]-1, \hat{\theta}_{y, i}[$.

7-4: $0<\widehat{\phi}_{y, i}<\hat{\theta}_{y, i}<1$ and $\left.\theta_{P, i} \in D_{\theta_{P, i}}=\right] \hat{\theta}_{y, i}, 1[$.

Proof: See Appendix 7.

Proposition 8: The NGF converge and they are upwardly and downwardly bounded such that:

8-1: $\forall \hat{A}>0$ we have $\left.\theta_{P, i} \in\right]-1, \hat{\theta}_{y, i}[$ and we get the following upper and lower bounds:

$0<\sigma_{\varepsilon, i}^{2}\left(\theta_{P, i}\right)<\hat{\sigma}_{\omega, i}^{2}\left(\frac{1+\widehat{\theta}_{y, i}}{1+\widehat{\phi}_{y, i}}\right)^{2} ;\left[\hat{\phi}_{y, i} \hat{V}_{1}+\left(\hat{\phi}_{y, i}^{2}-1\right) \hat{V}_{2}\right]\left(1+\widehat{\phi}_{y, i}\right)^{-2}<\sigma_{a, i}^{2}\left(\theta_{P, i}\right)<\hat{\sigma}_{\omega, i}^{2}$.

8-2: $\forall \hat{A}<0$ we have $\left.\theta_{P, i} \in\right] \widehat{\theta}_{y, i}, 1[$ and we get the following upper and lower bounds:

$0<\sigma_{\varepsilon, i}^{2}\left(\theta_{P, i}\right)<\hat{\sigma}_{\omega, i}^{2}\left(\frac{1-\hat{\theta}_{y, i}}{1-\widehat{\phi}_{y, i}}\right)^{2} ;-\left[\hat{\phi}_{y, i} \hat{V}_{1}+\left(\hat{\phi}_{y, i}^{2}-1\right) \hat{V}_{2}\right]\left(1-\hat{\phi}_{y, i}\right)^{-2}<\sigma_{a, i}^{2}\left(\theta_{P, i}\right)<\hat{\sigma}_{\omega, i}^{2}$.

Proof: See Appendix 8.

Empirically, the NGF will differ from a sample to another given that for each sample we get different values of the parameters $\hat{\phi}_{y, i}, \hat{\theta}_{y, i}$ and $\hat{\sigma}_{\omega, i}^{2}$. Although the NGF differ from a sample to another, they have to get the same fundamental characteristics as positiveness, convergence and bijection. In fact, the convergence of the NGF is a sine qua non condition for the forward foreign exchange market partial equilibrium. Namely, if the first source of noise, $\sigma_{\varepsilon, i}^{2}\left(\theta_{P, i}\right)$, is infinite, the demand as well as the supply function will be null and therefore the market mechanism will be truncated. Side by side, if the second source of noise, $\sigma_{a, i}^{2}\left(\theta_{P, i}\right)$, does not converge, the variance of the forward risk premium component will consequently diverge, and so does the variance of the demand as well as the variance of the supply. In spite the unidentified noise variances, we have identified upper and lower bounds that do not depend on the unknown parameter $\theta_{P, i}$, We have identified boudaries in a general $\operatorname{ARMA}(1,1)$ framework and the NGF converge for all $\theta_{P, i} \in D_{\theta_{P, i}}$ and therefore the unconditional unobserved forward risk premium variance, $\sigma_{P, i}^{2}\left(\theta_{P, i}\right)$, as well as the unconditional observed variance will converge. Furthermore, the noise variances are of opposite variation. A plausible question arises from the fact that the NGF covary in opposite directions: how do the signal 
variance and the first source of noise variance covary? It is easily shown that they negatively covary. From an empirical point of view, if one is willing to vary the unknown MA coefficient $\theta_{P, i}$ in order to minimize the signal variance, he should take into account that the noise variance, $\sigma_{\varepsilon, i}^{2}\left(\theta_{P, i}\right)$, will rise and vice versa. Indeed, the signal variance and the second source of noise variance, $\sigma_{a, i}^{2}\left(\theta_{P, i}\right)$, covary in the same direction with respect to the unknown MA coefficient $\theta_{P, i}$. It is essential to focus on the signal-to-noise ratio, the signal variance over the first source of noise variance, and to deepen insights on. In fact, if the underlying ratio is greater than one then more (less) than half of the time variation in forward exchange rates is explained by the time variation in forward risk premia components (by the time variation in noise). Otherwise, less (more) than half of the time variation in forward exchange rates is explained by the time variation in forward risk premia components (by the time variation in noise). The signal-to-noise ratio is of most importance within the signal extraction analysis. It sheds the light on the explanation of the time variation in the context of a signal plus noise model. This can be helpful when one is willing to simulate the unknown MA coefficient $\theta_{P, i}$. Another important ratio in the signal extraction-based approach is the second source of noise variance over the signal variance. It conveys us the information whether the time variation in the signal emanates essentially from the random components or not. In fact, if the ratio is greater than one half then more than half of the time variation in the signal is of a random nature. Otherwise, more than or exactly half of the time variation in the forward risk premia components emanates essentially from the systematic components.

Proposition 9:

9-1: $\left.\forall \theta_{P, i} \in\right] \widehat{\phi}_{y, i}-\left(1-\hat{\phi}_{y, i}^{2}\right)^{1 / 2}, \hat{\phi}_{y, i}+\left(1-\widehat{\phi}_{y, i}^{2}\right)^{1 / 2}[$, we get a ratio greater than .5.

9-2: $\left.\forall \theta_{P, i} \notin\right] \hat{\phi}_{y, i}-\left(1-\hat{\phi}_{y, i}^{2}\right)^{1 / 2}, \hat{\phi}_{y, i}+\left(1-\hat{\phi}_{y, i}^{2}\right)^{1 / 2}[$, we get a ratio less than one .5 .

9-3: $\forall \theta_{P, i}=\widehat{\phi}_{y, i}-\left(1-\hat{\phi}_{y, i}^{2}\right)^{1 / 2}$ or $\theta_{P, i}=\hat{\phi}_{y, i}+\left(1-\hat{\phi}_{y, i}^{2}\right)^{1 / 2}$, we get a ratio equal to .5 .

Proof: See Appendix 9.

Then we can base simulations on values taken by this ratio with respect to $1 / 2$. The simulations could be based upon prior informations concerning either the coefficient $\theta_{P, i}$ or the state of noise trading $\left(\sigma_{\varepsilon, i}^{2} \sigma_{a, i}^{2}\right)$.

5.2.2 $A R M A(2,2)$ as an Observable Model and $\operatorname{ARMA}(2,2)$ as a Hypothetical Model for the Signal

$\left(y_{t+1, i}=f_{t, 1, i}-S_{t+1, i}\right)$ follows an observable $\operatorname{ARMA}(2,2)$ model then we get the following RF:

$$
\left(1-\hat{\phi}_{1, y, i} B-\hat{\phi}_{2, y, i} B^{2}\right) y_{t+1, i}=\left(1-\hat{\theta}_{1, y, i} B-\hat{\theta}_{2, y, i} B^{2}\right) \omega_{t+1, i}
$$

The forward risk premium hypothetically follows an $A R M A(2,2)$ model then we obtain the following SF:

$$
\left(1-\hat{\phi}_{1, y, i} B-\hat{\phi}_{2, y, i} B^{2}\right) y_{t+1, i}=\left(1-\theta_{1, P, i} B-\theta_{2, P, i} B^{2}\right) a_{t, i}+\left(1-\hat{\phi}_{1, y, i} B-\hat{\phi}_{2, y, i} B^{2}\right) \varepsilon_{t+1, i}
$$

Now we calculate the unconditional variance, the first-order and second-order autocovariances for both the RF and $\mathrm{SF}$ and we get the following system of three equations and four unknowns:

$$
\begin{aligned}
& \text { System: } \quad \gamma_{0, S F}=\gamma_{0, R F} \\
& \gamma_{1, S F}=\gamma_{1, R F} \\
& \gamma_{2, S F}=\gamma_{2, R F} \\
& \text { System: } \quad g_{\widehat{\phi}_{1, y, i} ; \widehat{\phi}_{2, y, i}}\left(\theta_{1, P, i}, \theta_{2, P, i}\right) \sigma_{a, i}^{2}+\left(1-\hat{\phi}_{1, y, i}^{2}-\widehat{\phi}_{2, y, i}^{2}\right) \sigma_{\varepsilon, i}^{2}=\hat{A} \hat{\sigma}_{\omega, i}^{2} \\
& j_{\widehat{\phi}_{1, y, i} i \widehat{\phi}_{2, y, i}}\left(\theta_{1, P, i}, \theta_{2, P, i}\right) \sigma_{a, i}^{2}-\widehat{\phi}_{1, y, i} \hat{\phi}_{2, y, i} \sigma_{\varepsilon, i}^{2}=\hat{B} \hat{\sigma}_{\omega, i}^{2} \\
& l_{\widehat{\phi}_{1, y, i}, \hat{\phi}_{2, y, i}}\left(\theta_{1, P, i}, \theta_{2, P, i}\right) \sigma_{a, i}^{2}=\hat{C} \hat{\sigma}_{\omega, i}^{2}
\end{aligned}
$$

Where:

$$
\begin{aligned}
& g_{\widehat{\phi}_{1, y, i} ; \widehat{\phi}_{2, y, i}}\left(\theta_{1, P, i}, \theta_{2, P, i}\right)=\left(\theta_{1, P, i}-\widehat{\phi}_{1, y, i}\right)^{2}+\left(\theta_{2, P, i}-\hat{\phi}_{2, y, i}\right)^{2}+2 \hat{\phi}_{1, y, i} \theta_{2, P, i}\left(\theta_{1, P, i}-\hat{\phi}_{1, y, i}\right)+\left(1-\hat{\phi}_{1, y, i}^{2}-\hat{\phi}_{2, y, i}^{2}\right), \\
& j_{\widehat{\phi}_{1, y, i}, \hat{\phi}_{2, y, i}}\left(\theta_{1, P, i}, \theta_{2, P, i}\right)=\left(\theta_{1, P, i}-\hat{\phi}_{1, y, i}\right)\left[\hat{\phi}_{1, y, i} \theta_{1, P, i}+\theta_{2, P, i}\left(1+\hat{\phi}_{1, y, i}^{2}+\hat{\phi}_{2, y, i}\right)-1\right]+\hat{\phi}_{1, y, i} \theta_{2, P, i}\left(\theta_{2, P, i}-\right.
\end{aligned}
$$


$\left.\hat{\phi}_{2, y, i}\right)-\hat{\phi}_{2, y, i} \theta_{1, P, i}, \quad l_{\hat{\phi}_{1, y, i}, \widehat{\phi}_{2, y, i}}\left(\theta_{1, P, i}, \theta_{2, P, i}\right)=\left[\hat{\phi}_{1, y, i}\left(\theta_{1, P, i}-\hat{\phi}_{1, y, i}\right)+\left(\theta_{2, P, i}-\hat{\phi}_{2, y, i}\right)\right]\left[\hat{\phi}_{2, y, i} \theta_{2, P, i}+\hat{\phi}_{1, y, i} \theta_{1, P, i}+\right.$

$\left.\hat{\phi}_{1, y, i}^{2} \theta_{2, P, i}-1\right]+\left(\theta_{1, P, i}-\hat{\phi}_{1, y, i}\right)\left(\hat{\phi}_{2, y, i} \theta_{1, P, i}+\hat{\phi}_{1, y, i} \hat{\phi}_{2, y, i} \theta_{2, P, i}\right) \quad, \quad \hat{A}=g_{\widehat{\phi}_{1, y, i} i \widehat{\phi}_{2, y, i}}\left(\theta_{1, P, i}=\hat{\theta}_{1, y, i}, \theta_{2, P, i}=\hat{\theta}_{2, y, i}\right)$,

$\widehat{B}=j_{\widehat{\phi}_{1, y, i}, \widehat{\phi}_{2, y, i}}\left(\theta_{1, P, i}=\hat{\theta}_{1, y, i}, \theta_{2, P, i}=\hat{\theta}_{2, y, i}\right)$, and $\hat{C}=l_{\widehat{\phi}_{1, y, i}, \widehat{\phi}_{2, y, i}}\left(\theta_{1, P, i}=\hat{\theta}_{1, y, i}, \theta_{2, P, i}=\hat{\theta}_{2, y, i}\right)$.

The system of equations (14), (15), and (16) can be rewritten as follows:

System:

$$
g_{\widehat{\phi}_{1, y, i}, \widehat{\phi}_{2, y, i}}\left(\theta_{1, P, i}, \theta_{2, P, i}\right) \sigma_{a, i}^{2}+\left(1-\hat{\phi}_{1, y, i}^{2}-\hat{\phi}_{2, y, i}^{2}\right) \sigma_{\varepsilon, i}^{2}=\hat{A} \hat{\sigma}_{\omega, i}^{2}
$$

$$
\left[j_{\widehat{\phi}_{1, y, i} ;} \widehat{\phi}_{2, y, i}\left(\theta_{1, P, i}, \theta_{2, P, i}\right)+l_{\widehat{\phi}_{1, y, i} ; \widehat{\phi}_{2, y, i}}\left(\theta_{1, P, i}, \theta_{2, P, i}\right)\right] \sigma_{a, i}^{2}-\widehat{\phi}_{1, y, i} \hat{\phi}_{2, y, i} \sigma_{\varepsilon, i}^{2}=(\hat{B}+\hat{C}) \hat{\sigma}_{\omega, i}^{2}
$$

The system of equations (17) and (18) can be rewritten in a matrix form. We have:

$$
X_{\theta_{1, P, i}, \theta_{2, P, i}} . \Sigma_{N G F}=\hat{V}
$$

Where $X_{\theta_{1, P, i}, \theta_{2, P, i}}$ is a $2 \times 2$ matrix, $\Sigma_{N G F}$ is the $2 \times 1$ NGF vector, and $\hat{V}$ is a known $2 \times 1$ vector such that:

$$
\begin{gathered}
X_{\theta_{1, P, i}, \theta_{2, P, i}}=\left(\begin{array}{cc}
g_{\widehat{\phi}_{1, y, i}, \hat{\phi}_{2, y, i}}\left(\theta_{1, P, i}, \theta_{2, P, i}\right) & \left(1-\hat{\phi}_{1, y, i}^{2}-\hat{\phi}_{2, y, i}^{2}\right) \\
j_{\widehat{\phi}_{1, y, i}, \widehat{\phi}_{2, y, i}}\left(\theta_{1, P, i}, \theta_{2, P, i}\right)+l_{\widehat{\phi}_{1, y, i} i \widehat{\phi}_{2, y, i}}\left(\theta_{1, P, i}, \theta_{2, P, i}\right) & -\hat{\phi}_{1, y, i} \hat{\phi}_{2, y, i}
\end{array}\right) ; \Sigma_{N G F}=\left(\begin{array}{c}
\sigma_{a, i}^{2} \\
\sigma_{\varepsilon, i}^{2}
\end{array}\right) \text { and } \hat{V} \\
=\left(\begin{array}{c}
\hat{V}_{1} \\
\hat{V}_{2}
\end{array}\right)=\left(\begin{array}{c}
\hat{A} \hat{\sigma}_{\omega, i}^{2} \\
(\hat{B}+\hat{C}) \hat{\sigma}_{\omega, i}^{2}
\end{array}\right) .
\end{gathered}
$$

Pointing out the corresponding NGF is equivalent to solving equation (19) in $\Sigma_{\text {NGF. }}$ In fact, the eventual solution depends on the invertibility of the matrix $X_{\theta_{1, P, i}, \theta_{2, P, i}}$ and the conditions the NGF have to satisfy.

Proposition 10: $\forall \operatorname{det}\left(X_{\theta_{1, P, i}, \theta_{2, P, i}}\right)=d_{\widehat{\phi}_{1, y, i}, \widehat{\phi}_{2, y, i}}\left(\theta_{1, P, i}, \theta_{2, P, i}\right) \neq 0, X_{\theta_{1, P, i}, \theta_{2, P, i}}$ is nonsingular and NGF $\exists$, we get : $\Sigma_{N G F}\left(\theta_{1, P, i}, \theta_{2, P, i}\right)=X_{\theta_{1, P, i}, \theta_{2, P, i}}^{-1} . \hat{V}=$

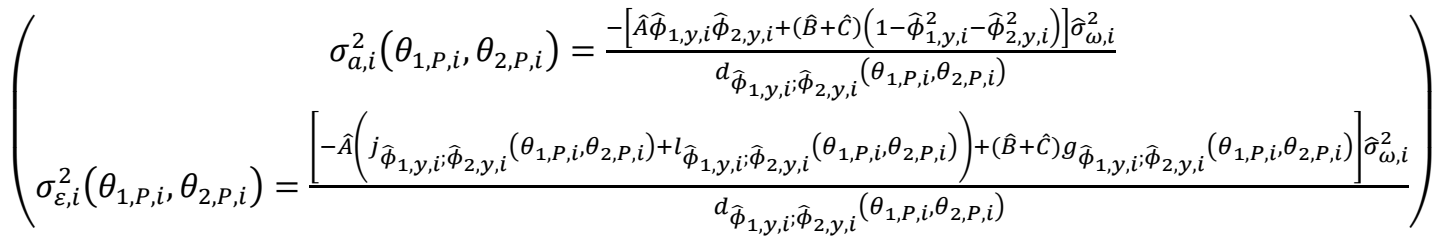

Proof: See Appendix 10.

The NGF have to be positive because they are variances. Thus it follows that the domain on which we define our NGF is the following:

$D_{N G F}=\left\{\left(\theta_{1, P, i}, \theta_{2, P, i}\right) \in\right]-1,1[\times]-1,1\left[/ d_{\widehat{\phi}_{1, y, i} i \widehat{\phi}_{2, y, i}}\left(\theta_{1, P, i}, \theta_{2, P, i}\right) \neq 0 \quad\right.$ and $\quad-\left[\hat{A} \hat{\phi}_{1, y, i} \hat{\phi}_{2, y, i}+(\hat{B}+\hat{C})(1-\right.$ $\left.\left.\hat{\phi}_{1, y, i}^{2}-\hat{\phi}_{2, y, i}^{2}\right)\right],\left[-\hat{A}\left(j_{\widehat{\phi}_{1, y, i}, \widehat{\phi}_{2, y, i}}\left(\theta_{1, P, i}, \theta_{2, P, i}\right)+l_{\widehat{\phi}_{1, y, i} i \widehat{\phi}_{2, y, i}}\left(\theta_{1, P, i}, \theta_{2, P, i}\right)\right)+(\hat{B}+\hat{C}) g_{\widehat{\phi}_{1, y, i}, \hat{\phi}_{2, y, i}}\left(\theta_{1, P, i}, \theta_{2, P, i}\right)\right]$ and $z_{\widehat{\phi}_{1, y, i}, \hat{\phi}_{2, y, i}}\left(\theta_{1, P, i}, \theta_{2, P, i}\right)$ have the same sign $\}$.

In addition, we easily show that the noise variances are upwardly bounded and the upper bounds are the maxima of the corresponding functions (Note 13). Furthermore, whenever we deal with an underdetermined system of equations, involving more than two equations, we reduce it to an equivalent system dealing with two equations as done above.

5.2.3 $A R(1)$ as an Observable Model and $\operatorname{ARMA}(1,1)$ as a Hypothetical Model for the Signal

$\left(y_{t+1, i}=f_{t, 1, i}-S_{t+1, i}\right)$ follows an observable $A R(1)$ model then we get the following RF:

$$
\left(1-\hat{\phi}_{y, i} B\right) y_{t+1, i}=\omega_{t+1, i}
$$

The forward risk premium hypothetically follows an $\operatorname{ARMA}(1,1)$ model then we obtain the following SF: 


$$
\left(1-\hat{\phi}_{y, i} B\right) y_{t+1, i}=\left(1-\theta_{P, i} B\right) a_{t, i}+\left(1-\hat{\phi}_{y, i} B\right) \varepsilon_{t+1, i}
$$

Now we calculate the unconditional variance and the first-order autocovariance for both the RF and SF and we get the following system of two equations and three unknowns:

$$
\begin{array}{cc}
\text { System: } & \gamma_{0, S F}=\gamma_{0, R F} \\
& \gamma_{1, S F}=\gamma_{1, R F} \\
\text { System: } & \left(\theta_{P, i}^{2}-2 \hat{\phi}_{y, i} \theta_{P, i}+1\right) \sigma_{a, i}^{2}+\left(1-\hat{\phi}_{y, i}^{2}\right) \sigma_{\varepsilon, i}^{2}=\hat{\sigma}_{\omega, i}^{2} \\
& \left(\hat{\phi}_{y, i}-\theta_{P, i}\right)\left(1-\hat{\phi}_{y, i} \theta_{P, i}\right) \sigma_{a, i}^{2}=\hat{\phi}_{y, i} \hat{\sigma}_{\omega, i}^{2}
\end{array}
$$

The system above can be rewritten in a matrix form. We have:

$$
X_{\theta_{P, i}} \Sigma_{N G F}=\widehat{V}
$$

Where $X_{\theta_{P, i}}$ is a $2 \times 2$ matrix, $\Sigma_{N G F}$ is the $2 \times 1$ NGF vector, and $\hat{V}$ is a known $2 \times 1$ vector such that:

$$
X_{\theta_{P, i}}=\left(\begin{array}{cc}
\theta_{P, i}^{2}-2 \hat{\phi}_{y, i} \theta_{P, i}+1 & 1-\hat{\phi}_{y, i}^{2} \\
\left(\hat{\phi}_{y, i}-\theta_{P, i}\right)\left(1-\hat{\phi}_{y, i} \theta_{P, i}\right) & 0
\end{array}\right) ; \Sigma_{N G F}=\left(\begin{array}{c}
\sigma_{a, i}^{2} \\
\sigma_{\varepsilon, i}^{2}
\end{array}\right) \text { and } \hat{V}=\left(\begin{array}{c}
\hat{V}_{1} \\
\hat{V}_{2}
\end{array}\right)=\left(\begin{array}{c}
\hat{\sigma}_{\omega, i}^{2} \\
\hat{\phi}_{y, i} \hat{\sigma}_{\omega, i}^{2}
\end{array}\right) \text {. }
$$

Pointing out the corresponding NGF is equivalent to solving equation (24) in $\Sigma_{\mathrm{NGF}}$. In fact, the eventual solution depends on the invertibility of the matrix $X_{\theta_{P, i}}$ and the conditions the NGF have to satisfy.

Proposition 11: $\left.\forall \theta_{P, i} \in\right]-1,1\left[\backslash\left\{\hat{\phi}_{y, i}\right\}, X_{\theta_{P, i}}\right.$ is nonsingular and NGF $\exists$, we get $\Sigma_{N G F}\left(\theta_{P, i}\right)=X_{\theta_{P, i}}^{-1} \cdot \hat{V}$ :

$\Sigma_{N G F}\left(\theta_{P, i}\right)=\left(\begin{array}{c}\sigma_{a, i}^{2}\left(\theta_{P, i}\right)=\hat{\phi}_{y, i}\left[\left(\hat{\phi}_{y, i}-\theta_{P, i}\right)\left(1-\hat{\phi}_{y, i} \theta_{P, i}\right)\right]^{-1} \hat{\sigma}_{\omega, i}^{2} \\ \sigma_{\varepsilon, i}^{2}\left(\theta_{P, i}\right)=\left[\left[\left(\hat{\phi}_{y, i}-\theta_{P, i}\right)\left(1-\hat{\phi}_{y, i} \theta_{P, i}\right)\right]^{-1}-\left[\frac{\widehat{\phi}_{y, i}\left(\theta_{P, i}^{2}-2 \widehat{\phi}_{y, i} \theta_{P, i}+1\right)}{\left(1-\widehat{\phi}_{y, i}^{2}\right)\left(\widehat{\phi}_{y, i}-\theta_{P, i}\right)\left(1-\widehat{\phi}_{y, i} \theta_{P, i}\right)}\right]\right]\end{array}\right)$.

Proof: See Appendix 11.

The domain on which we define our NGF is the following:

$D_{N G F}=\left\{\theta_{P, i} \in\right]-1,1\left[\backslash\left\{\hat{\phi}_{y, i}\right\} / \hat{\phi}_{y, i}\left[\left(\hat{\phi}_{y, i}-\theta_{P, i}\right)\left(1-\hat{\phi}_{y, i} \theta_{P, i}\right)\right]^{-1}>0 \quad\right.$ and $\quad\left[\left(\hat{\phi}_{y, i}-\theta_{P, i}\right)\left(1-\hat{\phi}_{y, i} \theta_{P, i}\right)\right]^{-1}-$ $\left.\left[\frac{\widehat{\phi}_{y, i}\left(\theta_{P, i}^{2}-2 \widehat{\phi}_{y, i} \theta_{P, i}+1\right)}{\left(1-\widehat{\phi}_{y, i}^{2}\right)\left(\widehat{\phi}_{y, i}-\theta_{P, i}\right)\left(1-\widehat{\phi}_{y, i} \theta_{P, i}\right)}\right]>0\right\}$.

The same reasoning, as in section 5.2.1, is applied for the positiveness of the NGF, the convergence, the determination of upper and lower bounds and the significantly useful signal-to-noise ratios.

5.2.4 $A R(2)$ as an Observable Model and $A R M A(2,2)$ as a Hypothetical Model for the Signal

$\left(y_{t+1, i}=f_{t, 1, i}-S_{t+1, i}\right)$ follows an observable $A R(2)$ model then we get the following RF:

$$
\left(1-\hat{\phi}_{1, y, i} B-\hat{\phi}_{2, y, i} B^{2}\right) y_{t+1, i}=\omega_{t+1, i}
$$

The forward risk premium hypothetically follows an $\operatorname{ARMA}(2,2)$ model then we obtain the following SF:

$$
\left(1-\hat{\phi}_{1, y, i} B-\hat{\phi}_{2, y, i} B^{2}\right) y_{t+1, i}=\left(1-\theta_{1, P, i} B-\theta_{2, P, i} B^{2}\right) a_{t, i}+\left(1-\hat{\phi}_{1, y, i} B-\hat{\phi}_{2, y, i} B^{2}\right) \varepsilon_{t+1, i}
$$

Now we calculate the unconditional variance, the first-order and second-order autocovariances for both the RF and $\mathrm{SF}$ and we get the following system of three equations and four unknowns:

System:

$$
\begin{aligned}
& \gamma_{0, S F}=\gamma_{0, R F} \\
& \gamma_{1, S F}=\gamma_{1, R F} \\
& \gamma_{2, S F}=\gamma_{2, R F}
\end{aligned}
$$

System:

$$
\begin{gathered}
x_{\widehat{\phi}_{1, y, i} i \widehat{\phi}_{2, y, i}}\left(\theta_{1, P, i}, \theta_{2, P, i}\right) \sigma_{a, i}^{2}+\left(1-\hat{\phi}_{1, y, i}^{2}-\widehat{\phi}_{2, y, i}^{2}\right) \sigma_{\varepsilon, i}^{2}=\hat{\sigma}_{\omega, i}^{2} \\
y_{\widehat{\phi}_{1, y, i}, \widehat{\phi}_{2, y, i}}\left(\theta_{1, P, i}, \theta_{2, P, i}\right) \sigma_{a, i}^{2}-\widehat{\phi}_{1, y, i} \hat{\phi}_{2, y, i} \sigma_{\varepsilon, i}^{2}=\hat{\phi}_{1, y, i} \hat{\sigma}_{\omega, i}^{2}
\end{gathered}
$$




$$
z_{\widehat{\phi}_{1, y, i} i} \widehat{\phi}_{2, y, i}\left(\theta_{1, P, i}, \theta_{2, P, i}\right) \sigma_{a, i}^{2}=\left(\hat{\phi}_{1, y, i}^{2}+\widehat{\phi}_{2, y, i}\right) \hat{\sigma}_{\omega, i}^{2}
$$

Where $\quad x_{\widehat{\phi}_{1, y, i} ; \hat{\phi}_{2, y, i}}\left(\theta_{1, P, i}, \theta_{2, P, i}\right)=\left[1+\theta_{1, P, i}^{2}+\theta_{2, P, i}^{2}-2\left[\hat{\phi}_{1, y, i} \theta_{1, P, i}+\hat{\phi}_{2, y, i} \theta_{2, P, i}+\hat{\phi}_{1, y, i} \theta_{2, P, i}\left(\hat{\phi}_{1, y, i}-\theta_{1, P, i}\right)\right]\right]$

$y_{\widehat{\phi}_{1, y, i}, \widehat{\phi}_{2, y, i}}\left(\theta_{1, P, i}, \theta_{2, P, i}\right)=$

$\left[\left(\hat{\phi}_{1, y, i}-\theta_{1, P, i}\right)\left[1-\hat{\phi}_{1, y, i} \theta_{1, P, i}-\theta_{2, P, i}\left(1+\hat{\phi}_{1, y, i}^{2}+\hat{\phi}_{2, y, i}\right)\right]-\hat{\phi}_{2, y, i} \theta_{1, P, i}-\hat{\phi}_{1, y, i} \theta_{2, P, i}\left(\hat{\phi}_{2, y, i}-\theta_{2, P, i}\right)\right] \quad, \quad$ and $z_{\widehat{\phi}_{1, y, i} ; \widehat{\phi}_{2, y, i}}\left(\theta_{1, P, i}, \theta_{2, P, i}\right)=$

$\left[\left(\hat{\phi}_{1, y, i}-\theta_{1, P, i}\right)\left(\hat{\phi}_{1, y, i}-\hat{\phi}_{2, y, i} \theta_{1, P, i}-\hat{\phi}_{1, y, i} \hat{\phi}_{2, y, i} \theta_{2, P, i}\right)+\left(\hat{\phi}_{2, y, i}-\theta_{2, P, i}\right)-\right.$

$\left.\left(\hat{\phi}_{1, y, i} \theta_{1, P, i}+\hat{\phi}_{2, y, i} \theta_{2, P, i}+\hat{\phi}_{1, y, i}^{2} \theta_{2, P, i}\right)\left[\widehat{\phi}_{1, y, i}\left(\hat{\phi}_{1, y, i}-\theta_{1, P, i}\right)+\left(\hat{\phi}_{2, y, i}-\theta_{2, P, i}\right)\right]\right]$.

The system of equations (27), (28), and (29) can be rewritten as follows:

System:

$$
x_{\widehat{\phi}_{1, y, i} ; \widehat{\phi}_{2, y, i}}\left(\theta_{1, P, i}, \theta_{2, P, i}\right) \sigma_{a, i}^{2}+\left(1-\hat{\phi}_{1, y, i}^{2}-\widehat{\phi}_{2, y, i}^{2}\right) \sigma_{\varepsilon, i}^{2}=\hat{\sigma}_{\omega, i}^{2}
$$

$$
\begin{gathered}
\left.\left[y_{\widehat{\phi}_{1, y, i} ; \widehat{\phi}_{2, y, i}\left(\theta_{1, P, i},\right.} \theta_{2, P, i}\right)+z_{\widehat{\phi}_{1, y, i} ; \widehat{\phi}_{2, y, i}}\left(\theta_{1, P, i}, \theta_{2, P, i}\right)\right] \sigma_{a, i}^{2}-\widehat{\phi}_{1, y, i} \hat{\phi}_{2, y, i} \sigma_{\varepsilon, i}^{2} \\
=\left(\hat{\phi}_{1, y, i}+\hat{\phi}_{1, y, i}^{2}+\hat{\phi}_{2, y, i}\right) \hat{\sigma}_{\omega, i}^{2}
\end{gathered}
$$

The system of equations (30) and (31) can be rewritten in a matrix form. We have:

$$
X_{\theta_{1, P, i}, \theta_{2, P, i}} . \Sigma_{N G F}=\hat{V}
$$

Where $X_{\theta_{1, P, i}, \theta_{2, P, i}}$ is a $2 \times 2$ matrix, $\Sigma_{N G F}$ is the $2 \times 1$ NGF vector, and $\hat{V}$ is a known $2 \times 1$ vector such that:

$$
\begin{gathered}
X_{\theta_{1, P, i}, \theta_{2, P, i}}=\left(\begin{array}{cc}
x_{\widehat{\phi}_{1, y, i} ; \widehat{\phi}_{2, y, i}}\left(\theta_{1, P, i}, \theta_{2, P, i}\right) & \left(1-\hat{\phi}_{1, y, i}^{2}-\hat{\phi}_{2, y, i}^{2}\right) \\
y_{\widehat{\phi}_{1, y, i} ;} \widehat{\phi}_{2, y, i}\left(\theta_{1, P, i}, \theta_{2, P, i}\right)+z_{\widehat{\phi}_{1, y, i} ; \widehat{\phi}_{2, y, i}}\left(\theta_{1, P, i}, \theta_{2, P, i}\right) & -\hat{\phi}_{1, y, i} \hat{\phi}_{2, y, i}
\end{array}\right) ; \Sigma_{N G F}=\left(\begin{array}{c}
\sigma_{a, i}^{2} \\
\sigma_{\varepsilon, i}^{2}
\end{array}\right) \text { and } \hat{V} \\
=\left(\begin{array}{c}
\widehat{V}_{1} \\
\hat{V}_{2}
\end{array}\right)=\left(\begin{array}{c}
\hat{\sigma}_{\omega, i}^{2} \\
\left(\hat{\phi}_{1, y, i}+\hat{\phi}_{1, y, i}^{2}+\hat{\phi}_{2, y, i}\right) \hat{\sigma}_{\omega, i}^{2}
\end{array}\right) .
\end{gathered}
$$

Pointing out the corresponding NGF is equivalent to solving equation (32) in $\Sigma_{\mathrm{NGF}}$. In fact, the eventual solution depends on the invertibility of the matrix $X_{\theta_{P, i}}$ and the conditions the NGF have to satisfy.

Proposition 12: $\forall \operatorname{det}\left(X_{\theta_{1, P, i}, \theta_{2, P, i}}\right)=d_{\widehat{\phi}_{1, y, i} ; \widehat{\phi}_{2, y, i}}\left(\theta_{1, P, i}, \theta_{2, P, i}\right) \neq 0, X_{\theta_{1, P, i}, \theta_{2, P, i}}$ is nonsingular and NGF $\exists$, we get: $\Sigma_{N G F}\left(\theta_{1, P, i}, \theta_{2, P, i}\right)=X_{\theta_{1, P, i}, \theta_{2, P, i}}^{-1} . \widehat{V}=$

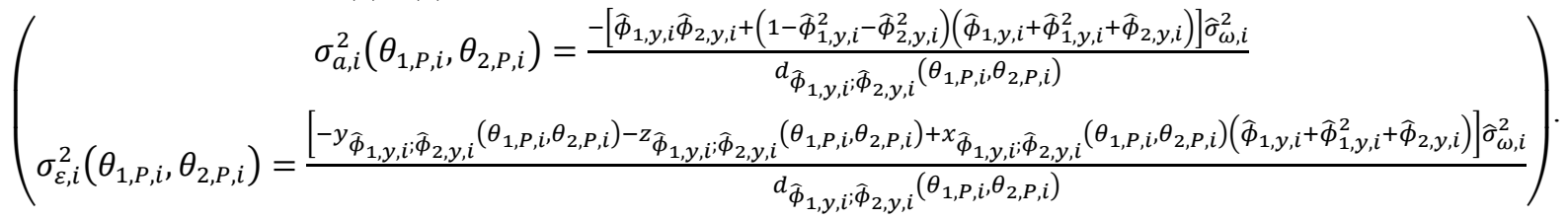

Proof: See Appendix 12.

The NGF have to be positive because they are variances. Thus it follows that the domain on which we define our NGF is the following:

$D_{N G F}=\left\{\left(\theta_{1, P, i}, \theta_{2, P, i}\right) \in\right]-1,1[\times]-1,1\left[/ d_{\widehat{\phi}_{1, y, i}, \hat{\phi}_{2, y, i}}\left(\theta_{1, P, i}, \theta_{2, P, i}\right) \neq 0 \quad\right.$ and $\quad \sigma_{a, i}^{2}\left(\theta_{1, P, i}, \theta_{2, P, i}\right)>0 \quad$ and $\left.\sigma_{\varepsilon, i}^{2}\left(\theta_{1, P, i}, \theta_{2, P, i}\right)>0\right\}$

In addition, we can easily show that the noise variances are upwardly bounded and the upper bounds are the maxima 
of the corresponding functions. Furthermore, whenever we deal with an underdetermined system of equations, involving more than two equations, we reduce it to an equivalent system dealing with two equations as done above.

5.2.5 MA(1) as an Observable Model and MA(1) as a Hypothetical Model for the Signal

$\left(y_{t+1, i}=f_{t, 1, i}-S_{t+1, i}\right)$ follows an observable MA(1) model then we get the following RF:

$$
y_{t+1, i}=\left(1-\hat{\theta}_{y, i} B\right) \omega_{t+1, i}
$$

The forward risk premium hypothetically follows a MA(1) model then we obtain the following SF:

$$
y_{t+1, i}=\left(1-\theta_{P, i} B\right) a_{t, i}+\varepsilon_{t+1, i}
$$

Now we calculate the unconditional variance and the first-order autocovariance for both the RF and SF and we get the following system of two equations and three unknowns:

$$
\begin{array}{cc}
\text { System: } & \gamma_{0, S F}=\gamma_{0, R F} \\
& \gamma_{1, S F}=\gamma_{1, R F} \\
\text { System: } & \left(1+\theta_{P, i}^{2}\right) \sigma_{a, i}^{2}+\sigma_{\varepsilon, i}^{2}=\left(1+\hat{\theta}_{y, i}^{2}\right) \hat{\sigma}_{\omega, i}^{2} \\
& \theta_{P, i} \sigma_{a, i}^{2}=\hat{\theta}_{y, i} \hat{\sigma}_{\omega, i}^{2}
\end{array}
$$

The system above can be rewritten in a matrix form. We have:

$$
X_{\theta_{P, i}}, \Sigma_{N G F}=\hat{V}
$$

Where $X_{\theta_{P, i}}$ is a $2 \times 2$ matrix, $\Sigma_{N G F}$ is the $2 \times 1$ NGF vector, and $\hat{V}$ is a known $2 \times 1$ vector such that:

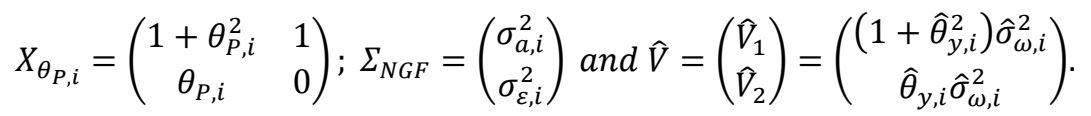

Pointing out the corresponding NGF is equivalent to solving equation (37) in $\Sigma_{\mathrm{NGF}}$. In fact, the eventual solution depends on the invertibility of the matrix $X_{\theta_{P, i}}$ and the conditions the NGF have to satisfy.

Proposition 13: $\left.\forall \theta_{P, i} \in\right]-1,1\left[\backslash\{0\}, X_{\theta_{P, i}}\right.$ is nonsingular and NGF $\exists$, we get:

$\Sigma_{N G F}\left(\theta_{P, i}\right)=X_{\theta_{P, i}}^{-1} \cdot \hat{V}=\left(\begin{array}{c}\sigma_{a, i}^{2}\left(\theta_{P, i}\right)=\hat{\theta}_{y, i} \hat{\sigma}_{\omega, i}^{2} \theta_{P, i}^{-1} \\ \sigma_{\varepsilon, i}^{2}\left(\theta_{P, i}\right)=\left[1+\hat{\theta}_{y, i}^{2}-\hat{\theta}_{y, i}\left(\frac{1+\theta_{P, i}^{2}}{\theta_{P, i}}\right)\right] \hat{\sigma}_{\omega, i}^{2}\end{array}\right)$.

Proof: See Appendix 13.

The domain on which we define our NGF is the following:

$D_{N G F}=\left\{\theta_{P, i} \in\right]-1,1\left[\backslash\{0\} / \hat{\theta}_{y, i} \hat{\sigma}_{\omega, i}^{2} \theta_{P, i}^{-1}>0\right.$ and $\left.\left[1+\hat{\theta}_{y, i}^{2}-\hat{\theta}_{y, i}\left(\frac{1+\theta_{P, i}^{2}}{\theta_{P, i}}\right)\right]>0\right\}$.

The same reasoning, as in section 5.2.1, is applied for the positiveness of the NGF, the convergence, the determination of upper and lower bounds and the significantly useful signal-to-noise ratios.

5.2.6 MA(2) as an Observable Model and MA(2) as a Hypothetical Model for the Signal

$\left(y_{t+1, i}=f_{t, 1, i}-S_{t+1, i}\right)$ follows an observable MA(2) model then we get the following RF:

$$
y_{t+1, i}=\left(1-\hat{\theta}_{1, y, i} B-\hat{\theta}_{2, y, i} B^{2}\right) \omega_{t+1, i}
$$

The forward risk premium hypothetically follows a $\mathrm{MA}(2)$ model then we obtain the following SF:

$$
y_{t+1, i}=\left(1-\theta_{1, P, i} B-\theta_{2, P, i} B^{2}\right) a_{t, i}+\varepsilon_{t+1, i}
$$

Now we calculate the unconditional variance, the first-order and second-order autocovariances for both the RF and $\mathrm{SF}$ and we get the following system of three equations and four unknowns:

$$
\begin{gathered}
\gamma_{0, S F}=\gamma_{0, R F} \\
\text { System: } \gamma_{1, S F}=\gamma_{1, R F} \\
\gamma_{2, S F}=\gamma_{2, R F} \\
\text { System: } \quad\left(1+\theta_{1, P, i}^{2}+\theta_{2, P, i}^{2}\right) \sigma_{a, i}^{2}+\sigma_{\varepsilon, i}^{2}=\left(1+\hat{\theta}_{1, y, i}^{2}+\hat{\theta}_{2, y, i}^{2}\right) \hat{\sigma}_{\omega, i}^{2} \\
\theta_{1, P, i}\left(1-\theta_{2, P, i}\right) \sigma_{a, i}^{2}=\hat{\theta}_{1, y, i}\left(1-\hat{\theta}_{2, y, i}\right) \hat{\sigma}_{\omega, i}^{2}
\end{gathered}
$$




$$
\theta_{2, P, i} \sigma_{a, i}^{2}=\hat{\theta}_{2, y, i} \hat{\sigma}_{\omega, i}^{2}
$$

The system of equations (40), (41), and (42) can be rewritten as follows:

System:

$$
\begin{gathered}
\left(1+\theta_{1, P, i}^{2}+\theta_{2, P, i}^{2}\right) \sigma_{a, i}^{2}+\sigma_{\varepsilon, i}^{2}=\left(1+\hat{\theta}_{1, y, i}^{2}+\hat{\theta}_{2, y, i}^{2}\right) \hat{\sigma}_{\omega, i}^{2} \\
{\left[\theta_{1, P, i}\left(1-\theta_{2, P, i}\right)+\theta_{2, P, i}\right] \sigma_{a, i}^{2}=\left[\hat{\theta}_{1, y, i}\left(1-\hat{\theta}_{2, y, i}\right)+\hat{\theta}_{2, y, i}\right] \hat{\sigma}_{\omega, i}^{2}}
\end{gathered}
$$

The system of equations (43) and (44) can be rewritten in a matrix form. We get:

$$
X_{\theta_{1, P, i}, \theta_{2, P, i}} . \Sigma_{N G F}=\hat{V}
$$

Where $X_{\theta_{1, P, i}, \theta_{2, P, i}}$ is a $2 \times 2$ matrix, $\Sigma_{N G F}$ is the $2 \times 1$ NGF vector, and $\widehat{V}$ is a known $2 \times 1$ vector such that:

$$
X_{\theta_{1, P, i}, \theta_{2, P, i}}=\left(\begin{array}{cc}
1+\theta_{1, P, i}^{2}+\theta_{2, P, i}^{2} & 1 \\
\theta_{1, P, i}\left(1-\theta_{2, P, i}\right)+\theta_{2, P, i} & 0
\end{array}\right) ; \Sigma_{N G F}=\left(\begin{array}{c}
\sigma_{a, i}^{2} \\
\sigma_{\varepsilon, i}^{2}
\end{array}\right) \text { and } \hat{V}=\left(\begin{array}{c}
\hat{V}_{1} \\
\hat{V}_{2}
\end{array}\right)=\left(\begin{array}{c}
\left(1+\hat{\theta}_{1, y, i}^{2}+\hat{\theta}_{2, y, i}^{2}\right) \hat{\sigma}_{\omega, i}^{2} \\
{\left[\hat{\theta}_{1, y, i}\left(1-\hat{\theta}_{2, y, i}\right)+\hat{\theta}_{2, y, i}\right] \hat{\sigma}_{\omega, i}^{2}}
\end{array}\right) \text {. }
$$

Pointing out the corresponding NGF is equivalent to solving equation (45) in $\Sigma_{\mathrm{NGF}}$. In fact, the eventual solution depends on the invertibility of the matrix $X_{\theta_{1, P, i}, \theta_{2, P, i}}$ and the conditions the NGF have to satisfy.

Proposition 14: $\left.\forall\left(\theta_{1, P, i}, \theta_{2, P, i}\right) \in\right]-1,1[\times]-1,1\left[/ \theta_{1, P, i} \neq-\frac{\theta_{2, P, i}}{1-\theta_{2, P, i}}\right.$ or $\theta_{2, P, i} \neq-\frac{\theta_{1, P, i}}{1-\theta_{1, P, i}}, X_{\theta_{1, P, i}, \theta_{2, P, i}}$ is invertible and NGF $\exists$, we get:

$\Sigma_{N G F}\left(\theta_{1, P, i}, \theta_{2, P, i}\right)=X_{\theta_{1, P, i}, \theta_{2, P, i}}^{-1} . \widehat{V}=$

$\left(\begin{array}{c}\sigma_{a, i}^{2}\left(\theta_{1, P, i}, \theta_{2, P, i}\right)=\left[\theta_{1, P, i}\left(1-\theta_{2, P, i}\right)+\theta_{2, P, i}\right]^{-1}\left[\hat{\theta}_{1, y, i}\left(1-\hat{\theta}_{2, y, i}\right)+\hat{\theta}_{2, y, i}\right] \hat{\sigma}_{\omega, i}^{2} \\ \sigma_{\varepsilon, i}^{2}\left(\theta_{1, P, i}, \theta_{2, P, i}\right)=\left[\left(1+\hat{\theta}_{1, y, i}^{2}+\hat{\theta}_{2, y, i}^{2}\right)-\left[\frac{\left(1+\theta_{1, P, i}^{2}+\theta_{2, P, i}^{2}\right)\left[\hat{\theta}_{1, y, i}\left(1-\hat{\theta}_{2, y, i}\right)+\hat{\theta}_{2, y, i}\right]}{\theta_{1, P, i}\left(1-\theta_{2, P, i}\right)+\theta_{2, P, i}}\right]\right] \hat{\sigma}_{\omega, i}^{2}\end{array}\right)$.

Proof: See Appendix 14.

$D_{N G F}=\left\{\left(\theta_{1, P, i}, \theta_{2, P, i}\right) \in\right]-1,1[\times]-1,1\left[/ \theta_{1, P, i} \neq-\frac{\theta_{2, P, i}}{1-\theta_{2, P, i}} \quad\right.$ or $\quad \theta_{2, P, i} \neq-\frac{\theta_{1, P, i}}{1-\theta_{1, P, i}} \quad$ and $\quad\left(1+\hat{\theta}_{1, y, i}^{2}+\hat{\theta}_{2, y, i}^{2}\right)>$ $\left.\left[\frac{\left(1+\theta_{1, P, i}^{2}+\theta_{2, P, i}^{2}\right)\left[\widehat{\theta}_{1, y, i}\left(1-\widehat{\theta}_{2, y, i}\right)+\widehat{\theta}_{2, y, i}\right]}{\theta_{1, P, i}\left(1-\theta_{2, P, i}\right)+\theta_{2, P, i}}\right]\right\}$.

The same reasoning, as in section 5.2.2 and 5.2.4, is applied for the positiveness of the NGF, the convergence, the determination of upper and lower bounds and the significantly useful signal-to-noise ratios.

\section{Conclusion}

Not for nothing do we pinpoint the underlying topic. Our paper proposes a synthesis of previously theoretical as well as empirical research and calls attention to a crucial problem, which is identifying an ARMA model for the unobserved forward risk premia. This paper focuses on the statistical problem of model identification for the unobservable forward risk premium component. We set up a theoretical framework to study the model identification problem for the signal. In fact, the true ARMA model for the unobserved signal could be either identifiable or unidentifiable. Indeed, the signal extraction approach is feasible only for the identifiable class of ARMA models for the forward risk premium signal buried in the noise. Otherwise, it is infeasible. Whenever an ARMA model for the signal is unidentifiable, we identify a new class of functions that we call: the NGF. They are noise variances expressed as functions of the unknown forward risk premium MA coefficients, they are bijective and they are upwardly and downwardly bounded. We mathematically show that an identifiable model for the forward risk premium component does not always exist and the signal extraction approach is not always feasible. As a matter of fact, the true model is not always the identifiable one. Thus, it is well-founded to deepen insight on the class of unidentifiable models which are described by the NGF. To apply our theoretical findings, we consider the empirically evidenced ARMA models within the related literature and we analyse each case pointing out its corresponding NGF.

\section{Acknowledgements}

We are grateful to several members of the BESTMOD (Business and Economic Statistics MODeling) lab, Institut Supérieur de Gestion, University of Tunis, for helpful comments and criticism.

\section{References}

Ansley, C.F., Spivey, W.A., \& Wrobleski, W.J. (1977). On The Structure of Moving Average Processes. Journal of 
Econometrics, 6, 121-134.

Bekaert, G., \& Hodrick, R.J. (1993). On Biases in The Measurement of Foreign Exchange Risk Premiums. Journal of International Money and Finance, 12, 115-138.

Bernoth, K., Von Hagen, J., \& De Vries, C.G. (2010). The Forward Premium Puzzle and Latent Factors Day by Day. Working Paper No 246/2010, De Nederlandsche Bank (DNB).

Bhar, R., \& Chiarella, C. (2009). Inference on Forward Exchange Rate Risk Premium: Reviewing Signal Extraction Methods. International Journal of Monetary Economics and Finance, 2(2), 115-125.

Bidarkota, P.V. (2004). Risk Premia in Forward Foreign Exchange Markets: A Comparison of Signal Extraction and Regression Methods. Working Paper No 0501, Florida International University, Department of Economics.

Boyer, R.S., \& Adams, F.C. (1988). Forward Premia and Risk Premia in a Simple Exchange Rate Model Determination. Journal of Money, Credit and Banking, 20(4), 633-644.

Cavaglia, S.M.F.G., Verschoor, W.F.C., \& Wolff, C.C.P. (1994). On The Biasedness of Forward Foreign Exchange Rqtes. Irrationality or Risk Premia?. Journal of Business, 67(3), 321-343.

Cheung, Y.W. (1993). Exchange Rate Risk Premiums. Journal of International Money and Finance, 12, $182-194$.

Diko, P., Lawford, S., \& Limpens, V. (2006). Risk Premia in Electricity forward Prices. Studies in Nonlinear Dynamics and Econometrics, 10, 3.

Djeutem, E. (2013). Model Uncertainty and the Forward Premium Puzzle. Working Paper, Simon Fraser University, $19^{\text {th }}$ International Conference of The Society for Computational Economics, Computing in Economics and finance.

Engel, C. (1996). The Forward Discount Anomaly and the Risk Premium: A Survey of Recent Evidence. Journal of Empirical Finance, 3, 123-192.

Fama, E.F. (1984). Forward and Spot Exchange Rates. Journal of Monetary Economics, 14, 319-338.

Fama, E.F. (1991). Efficient Capital Markets: II. The Journal of Finance, 46(5), 1575-1617.

Gospodinov, N. (2009). A New Look at the Forward Premium Puzzle. Journal of Financial Econometrics, 7(3), 312-338.

Isais-Torres, A.P., \& Cavazos-Cadena, R. (2012). Uniqueness of the Solution of the Yule-Walker Equations: A Vector Space Approach. Morfismos, 16(1), 29-43.

Jacobs, R.L. (1982). The Effect of Errors in Variables on Tests for a Risk Premium in Forward Exchange Rates. The Journal of Finance, 37(3), 667-677.

Nijman, T.E., Palm, F.C., \& Wolff, C.C.P. (1993). Premia in Forward Exchange Rates as Unobserved Components: A Note. Journal of Business and Economic Statistics, 11(3), 361-365.

Rezessy, A. (2010). Analysing Currency Risk Premia in The Czech Republic, Hungary, Poland and Slovakia. Working Paper No 2010/7, Magyar Nemzeti Bank (MNB).

Wolff, C.C.P. (1987). Forward Foreign Exchange Rates, Expected Spot Rates, and Premia: A Signal-Extraction Approach. The Journal of Finance, 42(2), 395-406.

Wolff, C.C.P. (2000). Measuring The Forward Foreign Exchange Risk Premium: Multi-Country Evidence from Unobserved Components Models. Journal of International Financial Markets, Institutions and Money, 10, 1-8.

\section{Notes}

Note 1. Forward premium anomaly.

Note 2. Martingale.

Note 3. Eugene Fama divided the work on informationally efficient capital markets into three categories: weak-form, semi-strong form, and strong-form tests which are replaced, respectively, in Fama (1991) by: tests for return forecastability, event studies, and private information tests.

Note 4. The ASW's theorem states that the summation of two uncorrelated moving average processes of orders $q_{2}$ and $\mathrm{q}_{3}$, respectively, has a $\operatorname{MA}\left(\mathrm{q}_{1}\right)$ representation such that $q_{1} \leq \operatorname{Max}\left(\mathrm{q}_{2}, \mathrm{q}_{3}\right)$.

Note 5 . The certainty equivalent is the level of wealth such that its utility is equal to the expected utility. 
Note 6. See Jacobs (1982).

Note 7. According to the existing empirical literature, we hypothesize that $\left(p_{3}, q_{3}\right)=(0,0)$ (i.e. a white noise).

Note 8. ASW's moving average summation theorem is relevant only for uncorrelated processes.

Note 9. $\left(a_{t, i}\right)$ is the second source of noise.

Note 10. Given an $\operatorname{ARMA}\left(p_{1}, q_{1}\right)$, we have $\gamma_{k} \neq 0$ for all $0 \leq k \leq \operatorname{Max}\left(p_{1}, q_{1}\right)$. Otherwise, $\gamma_{k}=0$.

Note 11. They are also called the Yule-Walker equations. As shown in Isais-Torres and Cavazos-Cadena (2012), theorem 3.1, the Yule-Walker matrix is invertible for all causal stochastic ARMA processes.

Note 12. The circumflex accent means that the coefficient is estimated and consequently known. An unknown coefficient will be written without a circumflex accent.

Note 13. We calculate the first partial derivatives and we deduce the stationary point by equalizing them to zero and we verify the second order conditions for maxima.

\section{Appendices}

Appendix 1. According to equation (4), the observed time series evidences the following $\operatorname{ARMA}\left(p_{1}, q_{1}\right)$ :

$$
\Phi_{y}(B) y_{t+1, i}=\Theta_{y}(B) \omega_{t+1, i}
$$

Where the polynomials $\Phi_{y}(B)$ and $\Theta_{y}(B)$ are known. Equation (4) is called the RF. In addition, we have:

$$
\begin{gathered}
\Phi_{P}(B) P_{t, 1, i}=\Theta_{P}(B) a_{t, i} \\
\Phi_{\varepsilon}(B) \varepsilon_{t+1, i}=\Theta_{\varepsilon}(B) v_{t+1, i}
\end{gathered}
$$

Combining equations (4), (46), and (47), we have the SF:

$$
\Phi_{\varepsilon}(B) \Phi_{P}(B) y_{t+1, i}=\Phi_{\varepsilon}(B) \Theta_{P}(B) a_{t, i}+\Phi_{P}(B) \Theta_{\varepsilon}(B) v_{t+1, i}
$$

In fact, a time series has a unique ARMA representation. Then equations (4) and (48), respectively the RF and the SF, are equivalent. It follows:

$$
\begin{gathered}
\Phi_{y}(B) y_{t+1, i}=\Phi_{\varepsilon}(B) \Phi_{P}(B) y_{t+1, i} \\
\Theta_{y}(B) \omega_{t+1, i}=\Phi_{\varepsilon}(B) \Theta_{P}(B) a_{t, i}+\Phi_{P}(B) \Theta_{\varepsilon}(B) v_{t+1, i}
\end{gathered}
$$

According to equation (49), we get

$$
\Phi_{y}(B)=\Phi_{\varepsilon}(B) \Phi_{P}(B)
$$

Straightforwardly, we have $\operatorname{deg}$ of $\Phi_{y}(B)=\operatorname{deg}$ of $\Phi_{\varepsilon}(B)+\operatorname{deg}$ of $\Phi_{P}(B) \Leftrightarrow p_{1}=p_{2}+p_{3}$. According to assumption 2 we have $p_{3}=0$ then we get $\Phi_{y}(B)=\Phi_{P}(B)$. It follows::

$$
p_{1}=p_{2}
$$

Now we apply ASW's theorem to equation (50). $\Theta_{y}(B) \omega_{t+1, i}$ is a $M A\left(q_{1}\right), \Phi_{\varepsilon}(B) \Theta_{P}(B) a_{t, i}$ is a $M A\left(q_{3}+p_{2}\right)$ and $\Phi_{P}(B) \Theta_{\varepsilon}(B) v_{t+1, i}$ is a $M A\left(q_{2}+p_{3}\right)$. According to assumption 2 we have $p_{3}=q_{3}=0$. Then $\Phi_{\varepsilon}(B) \Theta_{P}(B) a_{t, i}$ is a $M A\left(p_{2}\right)$ and $\Phi_{P}(B) \Theta_{\varepsilon}(B) v_{t+1, i}$ is a $M A\left(q_{2}\right)$. It follows:

$$
q_{1} \leq \operatorname{Max}\left(q_{2}, p_{2}=p_{1}\right)
$$

Finally, equation (52) and inequation (53) let us deduce that the unobservable forward risk premium, $P_{t, 1, i}$, follows an $\operatorname{ARMA}\left(p_{1}, q_{1}\right)$ such that: $p_{1}=p_{2}$ and $q_{1} \leq \operatorname{Max}\left(q_{2}, p_{1}\right)$. Q.E.D

Appendix 2. We have $\operatorname{Max}\left(p_{1}, q_{1}\right)+1$ linearly independent equations and the only unknown polynomial is $\Theta_{P}(B)$ which is of degree $q_{2}$, i.e. $\Theta_{P}(B)$ has $q_{2}$ unknown MA coefficients. In addition, $\sigma_{\varepsilon}^{2}$ and $\sigma_{a}^{2}$ are unknown. It follows that we have $q_{2}+2$ unknowns. The system has a unique solution, i.e. All unknowns are identified and each one gets a unique value, if the number of equations is equal to the number of unknowns: $q_{2}+2=\operatorname{Max}\left(p_{1}, q_{1}\right)+$ $1 \Leftrightarrow q_{2}=\operatorname{Max}\left(p_{1}, q_{1}\right)-1$. Q.E.D

Appendix 3. If the number of unknowns is greater than the number of equations then we get an underdetermined system of equations and we can express some unknowns as functions of others, so we get infinitely many solutions. On the other hand, if the number of equations is greater than the number of unknowns, then we have an overdetermined system, given that all equations are linearly independent, we have no solutions and the system of equations is inconsistent.

Appendix 4. We apply the proposition 1 so we get the proposition 4. 
Appendix 5. We apply the proposition 1 so we get the proposition 5.

Appendix 6. We have $\operatorname{det}\left(X_{\theta_{P, i}}\right)=d_{\widehat{\phi}_{y, i}}\left(\theta_{P, i}\right)=\hat{\phi}_{y, i} \theta_{P, i}^{2}-\left(1+\hat{\phi}_{y, i}^{2}\right) \theta_{P, i}+\hat{\phi}_{y, i}$.

$\Delta=\left(1-\hat{\phi}_{y, i}^{2}\right)^{2}$, so we get two roots: $\hat{\phi}_{y, i}$ and $\left.\hat{\phi}_{y, i}{ }^{-1} \cdot \hat{\phi}_{y, i}{ }^{-1} \notin\right]-1,1\left[\right.$ so we exclude it. Then $\operatorname{det}\left(X_{\theta_{P, i}}\right) \neq 0$ iif $\left.\theta_{P, i} \in\right]-1,1\left[\left\{\hat{\phi}_{y, i}\right.\right.$ and we get $\Sigma_{N G F}\left(\theta_{P, i}\right)=X_{\theta_{P, i}}^{-1} \hat{V}=X_{\theta_{P, i}}^{-1} \cdot\left(\begin{array}{l}\hat{V}_{1} \\ \hat{V}_{2}\end{array}\right) \cdot X_{\theta_{P, i}}^{-1}=\frac{1}{\operatorname{det}\left(x_{\theta_{P, i}}\right)}\left(\begin{array}{cc}\hat{\phi}_{y, i} & -\left(1-\hat{\phi}_{y, i}^{2}\right) \\ -\theta_{P, i} & \theta_{P, i}^{2}-2 \hat{\phi}_{y, i} \theta_{P, i}+1\end{array}\right)$ so we deduce that: $\Sigma_{N G F}\left(\theta_{P, i}\right)=\left(\begin{array}{c}\sigma_{a, i}^{2}\left(\theta_{P, i}\right)=\frac{\widehat{\phi}_{y, i} \widehat{V}_{1}+\left(\widehat{\phi}_{y, i}^{2}-1\right) \widehat{V}_{2}}{d_{\widehat{\phi}_{y, i}}\left(\theta_{P, i}\right)} \\ \sigma_{\varepsilon, i}^{2}\left(\theta_{P, i}\right)=\hat{\sigma}_{\omega, i}^{2} \frac{h_{\widehat{\theta}_{y, i}}\left(\theta_{P, i}\right)}{d_{\widehat{\phi}_{y, i}}\left(\theta_{P, i}\right)}\end{array}\right)$. Q.E.D

Appendix 7. We have $\sigma_{a, i}^{2}\left(\theta_{P, i}\right)=\frac{\hat{A}}{d_{\widehat{\phi}_{y, i}}\left(\theta_{P, i}\right)}>0$ and $\sigma_{\varepsilon, i}^{2}\left(\theta_{P, i}\right)=\hat{\sigma}_{\omega, i}^{2} \frac{h_{\widehat{\theta}_{y, i}}\left(\theta_{P, i}\right)}{d_{\widehat{\phi}_{y, i}}\left(\theta_{P, i}\right)}>0$. It follows that $\hat{A}=\hat{\phi}_{y, i} \widehat{V}_{1}+$ $\left(\hat{\phi}_{y, i}^{2}-1\right) \hat{V}_{2}=\hat{\phi}_{y, i} \hat{\theta}_{y, i}^{2}-\left(1+\hat{\phi}_{y, i}^{2}\right) \hat{\theta}_{y, i}+\hat{\phi}_{y, i}, d_{\widehat{\phi}_{y, i}}\left(\theta_{P, i}\right)$ and $h_{\hat{\theta}_{y, i}}\left(\theta_{P, i}\right)=\hat{\theta}_{y, i} \theta_{P, i}^{2}-\left(1+\hat{\theta}_{y, i}^{2}\right) \theta_{P, i}+\hat{\theta}_{y, i}$ must have the same sign. We have:

$\left.\hat{A}>0 \Leftrightarrow \hat{\theta}_{y, i} \in\right]-1, \hat{\phi}_{y, i}\left[, \hat{A}<0 \Leftrightarrow \hat{\theta}_{y, i} \in\right] \hat{\phi}_{y, i}, 1[$.

$\left.d_{\widehat{\phi}_{y, i}}\left(\theta_{P, i}\right)>0 \Leftrightarrow \theta_{P, i} \in\right]-1, \hat{\phi}_{y, i}\left[, d_{\widehat{\phi}_{y, i}}\left(\theta_{P, i}\right)<0 \Leftrightarrow \theta_{P, i} \in\right] \hat{\phi}_{y, i}, 1[$.

$\left.h_{\widehat{\theta}_{y, i}}\left(\theta_{P, i}\right)>0 \Leftrightarrow \theta_{P, i} \in\right]-1, \hat{\theta}_{y, i}\left[, h_{\widehat{\theta}_{y, i}}\left(\theta_{P, i}\right)<0 \Leftrightarrow \theta_{P, i} \in\right] \hat{\theta}_{y, i}, 1[$.

Four cases are consequently inferred:

Case 1: $\hat{A}>0, d_{\widehat{\phi}_{y, i}}\left(\theta_{P, i}\right)>0$, and $h_{\hat{\theta}_{y, i}}\left(\theta_{P, i}\right)>0$ such that $\left.\hat{\phi}_{y, i} \in\right]-1,0[$.

In fact, we have $\left.\hat{A}>0 \forall-1<\hat{\theta}_{y, i}<\widehat{\phi}_{y, i}<0, d_{\widehat{\phi}_{y, i}}\left(\theta_{P, i}\right)>0 \forall \theta_{P, i} \in\right]-1, \widehat{\phi}_{y, i}\left[\right.$, and $h_{\hat{\theta}_{y, i}}\left(\theta_{P, i}\right)>0 \forall \theta_{P, i} \in$ ]$-1, \hat{\theta}_{y, i}\left[\right.$. Given that $\hat{\theta}_{y, i}<\hat{\phi}_{y, i}$ we have $]-1, \hat{\theta}_{y, i}[\subset]-1, \hat{\phi}_{y, i}[$ then our domain is $]-1, \hat{\theta}_{y, i}[$. Q.E.D

Case 2: $\hat{A}<0, d_{\widehat{\phi}_{y, i}}\left(\theta_{P, i}\right)<0$, and $h_{\widehat{\theta}_{y, i}}\left(\theta_{P, i}\right)<0$ such that $\left.\hat{\phi}_{y, i} \in\right]-1,0[$.

In fact, we have $\hat{A}<0 \forall-1<\widehat{\phi}_{y, i}<\hat{\theta}_{y, i}<0$ or $\left.-1<\hat{\phi}_{y, i}<0<\hat{\theta}_{y, i}<1, d_{\hat{\phi}_{y, i}}\left(\theta_{P, i}\right)<0 \forall \theta_{P, i} \in\right] \hat{\phi}_{y, i}, 1[$, and $\left.h_{\widehat{\theta}_{y, i}}\left(\theta_{P, i}\right)<0 \forall \theta_{P, i} \in\right] \hat{\theta}_{y, i}, 1\left[\right.$. Given that $\hat{\theta}_{y, i}>\hat{\phi}_{y, i}$ we have $] \hat{\theta}_{y, i}, 1[\subset] \hat{\phi}_{y, i}, 1[$ then our domain is ]$\hat{\theta}_{y, i}, 1[$. Q.E.D

Case 3: $\hat{A}>0, d_{\hat{\phi}_{y, i}}\left(\theta_{P, i}\right)>0$, and $h_{\hat{\theta}_{y, i}}\left(\theta_{P, i}\right)>0$ such that $\left.\hat{\phi}_{y, i} \in\right] 0,1[$.

In fact, we have $\hat{A}>0 \forall-1<\hat{\theta}_{y, i}<0<\hat{\phi}_{y, i}<1$ or $\left.0<\hat{\theta}_{y, i}<\hat{\phi}_{y, i}<1, d_{\widehat{\phi}_{y, i}}\left(\theta_{P, i}\right)>0 \forall \theta_{P, i} \in\right]-1, \hat{\phi}_{y, i}[$, and $\left.h_{\widehat{\theta}_{y, i}}\left(\theta_{P, i}\right)>0 \forall \theta_{P, i} \in\right]-1, \hat{\theta}_{y, i}\left[\right.$. Given that $\hat{\theta}_{y, i}<\hat{\phi}_{y, i}$ we have $]-1, \hat{\theta}_{y, i}[\subset]-1, \hat{\phi}_{y, i}[$ then our domain is ]$-1, \hat{\theta}_{y, i}[$. Q.E.D

Case 4: $\hat{A}<0, d_{\widehat{\phi}_{y, i}}\left(\theta_{P, i}\right)<0$, and $h_{\widehat{\theta}_{y, i}}\left(\theta_{P, i}\right)<0$ such that $\left.\hat{\phi}_{y, i} \in\right] 0,1[$.

In fact, we have $\left.\hat{A}<0 \forall 0<\hat{\phi}_{y, i}<\hat{\theta}_{y, i}<1, d_{\widehat{\phi}_{y, i}}\left(\theta_{P, i}\right)<0 \forall \theta_{P, i} \in\right] \hat{\phi}_{y, i}, 1\left[\right.$, and $\left.h_{\widehat{\theta}_{y, i}}\left(\theta_{P, i}\right)<0 \forall \theta_{P, i} \in\right] \hat{\theta}_{y, i}, 1[$.

Given that $\hat{\theta}_{y, i}>\hat{\phi}_{y, i}$ we have $] \hat{\theta}_{y, i}, 1[\subset] \hat{\phi}_{y, i}, 1[$ then our domain is $] \hat{\theta}_{y, i}, 1[$. Q.E.D Appendix 8. We get two cases: 
Case 1: $\hat{A}>0$ then $\left.\theta_{P, i} \in\right]-1, \hat{\theta}_{y, i}\left[\right.$. We calculate the limits of the NGF when $\theta_{P, i}$ approaches $-1^{+}$and $\theta_{P, i}$ approaches $\hat{\theta}_{y, i}^{-}$. The former will give us the upper bound and the latter will give us the lower bound for $\sigma_{\varepsilon, i}^{2}\left(\theta_{P, i}\right)$ because it is a decreasing function on that domain. Whereas the former will give us the lower bound and the latter will give us the upper bound for $\sigma_{a, i}^{2}\left(\theta_{P, i}\right)$ because it is an increasing function on that domain. Q.E.D

Case 2: $\hat{A}<0$ then $\left.\theta_{P, i} \in\right] \hat{\theta}_{y, i}, 1\left[\right.$. We calculate the limits of the NGF when $\theta_{P, i}$ approaches $\hat{\theta}_{y, i}^{+}$and $\theta_{P, i}$ approaches $1^{-}$. The former will give us the upper bound and the latter will give us the lower bound for $\sigma_{a, i}^{2}\left(\theta_{P, i}\right)$ because it is a decreasing function on that domain. Whereas the former will give us the lower bound and the latter will give us the upper bound for $\sigma_{\varepsilon, i}^{2}\left(\theta_{P, i}\right)$ because it is an increasing function on that domain. Q.E.D

Appendix 9. Let $R\left(\theta_{P, i}\right)$ be $\frac{\sigma_{a, i}^{2}\left(\theta_{P, i}\right)}{\sigma_{P, i}^{2}\left(\theta_{P, i}\right)}$. We have $R\left(\theta_{P, i}\right)=\frac{1-\widehat{\phi}_{y, i}^{2}}{\theta_{P, i}^{2}-2 \widehat{\phi}_{y, i} \theta_{P, i}+1}$. Substracting one half from both sides, we get $R\left(\theta_{P, i}\right)-.5=-\frac{\theta_{P, i}^{2}-2 \widehat{\phi}_{y, i} \theta_{P, i}-\left(1-2 \widehat{\phi}_{y, i}^{2}\right)}{2\left(\theta_{P, i}^{2}-2 \widehat{\phi}_{y, i} \theta_{P, i}+1\right)}$. The denominator is always positive and different from zero. As a matter of fact $R\left(\theta_{P, i}\right)-.5$ has the opposite sign of the numerator. The roots of the numerator are $\hat{\phi}_{y, i}-\left(1-\hat{\phi}_{y, i}^{2}\right)^{1 / 2}$ and $\hat{\phi}_{y, i}+\left(1-\hat{\phi}_{y, i}^{2}\right)^{1 / 2}$ then it is negative $\left.\forall \theta_{P, i} \in\right] \hat{\phi}_{y, i}-\left(1-\hat{\phi}_{y, i}^{2}\right)^{1 / 2}, \hat{\phi}_{y, i}+\left(1-\hat{\phi}_{y, i}^{2}\right)^{1 / 2}[$ and positive $\left.\forall \theta_{P, i} \notin\right] \hat{\phi}_{y, i}-\left(1-\hat{\phi}_{y, i}^{2}\right)^{1 / 2}, \hat{\phi}_{y, i}+\left(1-\hat{\phi}_{y, i}^{2}\right)^{1 / 2}\left[\right.$. Consequently, we get $R\left(\theta_{P, i}\right)-.5$ is positive $\left.\forall \theta_{P, i} \in\right] \hat{\phi}_{y, i}-$ $\left(1-\hat{\phi}_{y, i}^{2}\right)^{1 / 2}, \hat{\phi}_{y, i}+\left(1-\hat{\phi}_{y, i}^{2}\right)^{1 / 2}\left[, \quad\right.$ negative $\left.\forall \theta_{P, i} \notin\right] \widehat{\phi}_{y, i}-\left(1-\hat{\phi}_{y, i}^{2}\right)^{1 / 2}, \hat{\phi}_{y, i}+\left(1-\hat{\phi}_{y, i}^{2}\right)^{1 / 2}[, \quad$ and $\quad$ null whenever $\theta_{P, i}$ equals the roots. Q.E.D

Appendix 10. $X_{\theta_{1, P, i}, \theta_{2, P, i}}$ is nonsingular and NGF $\exists$ iif $\operatorname{det}\left(X_{\theta_{1, P, i}, \theta_{2, P, i}}\right)=d_{\widehat{\phi}_{1, y, i} ; \bar{\phi}_{2, y, i}}\left(\theta_{1, P, i}, \theta_{2, P, i}\right) \neq 0$. $\forall d_{\widehat{\phi}_{1, y, i}, \widehat{\phi}_{2, y, i}}\left(\theta_{1, P, i}, \theta_{2, P, i}\right) \neq 0 \quad$ we $\quad$ get : $\quad \Sigma_{N G F}\left(\theta_{1, P, i}, \theta_{2, P, i}\right)=X_{\theta_{1, P, i}, \theta_{2, P, i}}^{-1} . \widehat{V} \quad$ such $\quad$ that $X_{\theta_{1, P, i}, \theta_{2, P, i}}^{-1}=$ $\frac{1}{d_{\widehat{\phi}_{1, y, i}, \hat{\phi}_{2, y, i}}\left(\theta_{1, P, i}, \theta_{2, P, i}\right)}\left(\begin{array}{cc}-\widehat{\phi}_{1, y, i} \hat{\phi}_{2, y, i} & -\left(1-\widehat{\phi}_{1, y, i}^{2}-\widehat{\phi}_{2, y, i}^{2}\right) \\ -\left(j_{\widehat{\phi}_{1, y, i} i \widehat{\phi}_{2, y, i}}\left(\theta_{1, P, i}, \theta_{2, P, i}\right)+l_{\widehat{\phi}_{1, y, i}, \widehat{\phi}_{2, y, i}}\left(\theta_{1, P, i}, \theta_{2, P, i}\right)\right) & g_{\widehat{\phi}_{1, y, i}, \widehat{\phi}_{2, y, i}}\left(\theta_{1, P, i}, \theta_{2, P, i}\right)\end{array}\right)$. It follows: $\Sigma_{N G F}\left(\theta_{1, P, i}, \theta_{2, P, i}\right)=X_{\theta_{1, P, i}, \theta_{2, P, i}}^{-1} \cdot \hat{V}=X_{\theta_{1, P}, i}^{-1} \theta_{2, P, i} \cdot\left(\begin{array}{c}\hat{A} \hat{\sigma}_{\omega, i}^{2} \\ (\hat{B}+\hat{C}) \hat{\sigma}_{\omega, i}^{2}\end{array}\right)$. Straightforwardly, we get: $\Sigma_{N G F}\left(\theta_{1, P, i}, \theta_{2, P, i}\right)=$

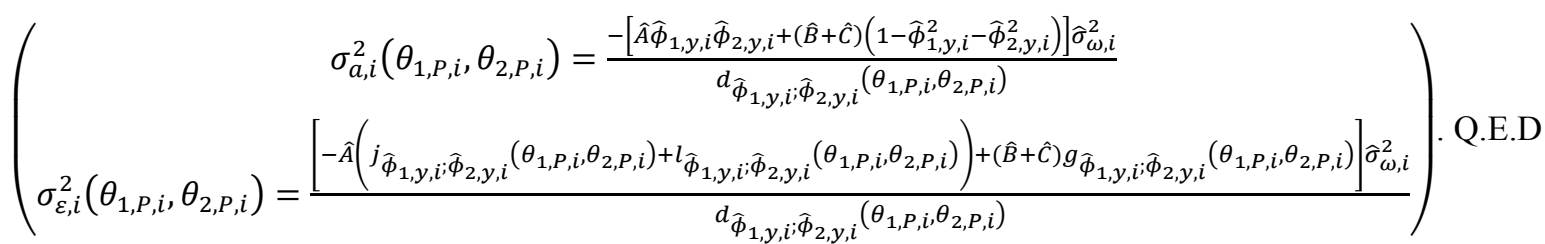

Appendix 11. The same reasoning as in the proof of proposition 10. Appendix 12. The same reasoning as in the proof of proposition 10. Appendix 13. The same reasoning as in the proof of proposition 10. Appendix 14. The same reasoning as in the proof of proposition 10 . 